\title{
Solvability and Algorithms for Functional Equations Originating from Dynamic Programming
}

\author{
Guojing Jiang, ${ }^{1}$ Shin Min Kang, ${ }^{2}$ and Young Chel Kwun ${ }^{3}$ \\ ${ }^{1}$ Organization Department, Dalian Vocational Technical College, Dalian, Liaoning 116035, China \\ 2 Department of Mathematics and RINS, Gyeongsang National University, Jinju 660-701, Republic of Korea \\ ${ }^{3}$ Department of Mathematics, Dong-A University, Pusan 614-714, Republic of Korea
}

Correspondence should be addressed to Young Chel Kwun, yckwun@dau.ac.kr

Received 5 January 2011; Accepted 11 February 2011

Academic Editor: Yeol J. Cho

Copyright (C) 2011 Guojing Jiang et al. This is an open access article distributed under the Creative Commons Attribution License, which permits unrestricted use, distribution, and reproduction in any medium, provided the original work is properly cited.

The main purpose of this paper is to study the functional equation arising in dynamic programming of multistage decision processes $f(x)=$ opt $_{y \in D} \operatorname{opt}\{p(x, y), q(x, y) f(a(x, y)), r(x, y) f(b(x, y))$, $s(x, y) f(c(x, y))\}$ for all $x \in S$. A few iterative algorithms for solving the functional equation are suggested. The existence, uniqueness and iterative approximations of solutions for the functional equation are discussed in the Banach spaces $B C(S)$ and $B(S)$ and the complete metric space $B B(S)$, respectively. The properties of solutions, nonnegative solutions, and nonpositive solutions and the convergence of iterative algorithms for the functional equation and other functional equations, which are special cases of the above functional equation, are investigated in the complete metric space $B B(S)$, respectively. Eight nontrivial examples which dwell upon the importance of the results in this paper are also given.

\section{Introduction}

The existence, uniqueness, and iterative approximations of solutions for several classes of functional equations arising in dynamic programming were studied by a lot of researchers; see [1-23] and the references therein. Bellman [3], Bhakta and Choudhury [7], Liu [12], Liu and Kang [15], and Liu et al. [18] investigated the following functional equations:

$$
\begin{gathered}
f(x)=\inf _{y \in D} \max \{p(x, y), f(a(x, y))\}, \quad \forall x \in S, \\
f(x)=\sup _{y \in D} \max \{p(x, y), f(a(x, y))\}, \quad \forall x \in S, \\
f(x)=\inf _{y \in D} \max \{p(x, y), q(x, y) f(a(x, y))\}, \quad \forall x \in S
\end{gathered}
$$


and gave some existence and uniqueness results and iterative approximations of solutions for the functional equations in $B B(S)$. Liu and Kang [14] and Liu and Ume [17] generalized the results in $[3,7,12,15,18]$ and studied the properties of solutions, nonpositive solutions and nonnegative solutions and the convergence of iterative approximations for the following functional equations, respectively

$$
\begin{gathered}
f(x)=\underset{y \in D}{\operatorname{opt} \max }\{p(x, y), f(a(x, y))\}, \quad \forall x \in S, \\
f(x)=\underset{y \in D}{\operatorname{opt} \min }\{p(x, y), f(a(x, y))\}, \quad \forall x \in S, \\
f(x)=\underset{y \in D}{\operatorname{opt} \max }\{p(x, y), q(x, y) f(a(x, y))\}, \quad \forall x \in S, \\
f(x)=\underset{y \in D}{\operatorname{opt} \min }\{p(x, y), q(x, y) f(a(x, y))\}, \quad \forall x \in S
\end{gathered}
$$

in $B B(S)$.

The purpose of this paper is to introduce and study the following functional equations arising in dynamic programming of multistage decision processes:

$$
f(x)=\underset{y \in D}{\operatorname{opt} \operatorname{opt}}\{p(x, y), q(x, y) f(a(x, y)), r(x, y) f(b(x, y)), s(x, y) f(c(x, y))\}, \quad \forall x \in S,
$$

$$
f(x)=\underset{y \in D}{\operatorname{opt} \max }\{p(x, y), q(x, y) f(a(x, y)), r(x, y) f(b(x, y)), s(x, y) f(c(x, y))\}, \quad \forall x \in S,
$$

$$
f(x)=\underset{y \in D}{\operatorname{opt} \min }\{p(x, y), q(x, y) f(a(x, y)), r(x, y) f(b(x, y)), s(x, y) f(c(x, y))\}, \quad \forall x \in S,
$$

$$
f(x)=\sup _{y \in D} \max \{p(x, y), q(x, y) f(a(x, y)), r(x, y) f(b(x, y)), s(x, y) f(c(x, y))\}, \quad \forall x \in S,
$$

$$
f(x)=\inf _{y \in D} \min \{p(x, y), q(x, y) f(a(x, y)), r(x, y) f(b(x, y)), s(x, y) f(c(x, y))\}, \quad \forall x \in S,
$$

$$
f(x)=\sup _{y \in D} \min \{p(x, y), q(x, y) f(a(x, y)), r(x, y) f(b(x, y)), s(x, y) f(c(x, y))\}, \quad \forall x \in S,
$$

$$
f(x)=\inf _{y \in D} \max \{p(x, y), q(x, y) f(a(x, y)), r(x, y) f(b(x, y)), s(x, y) f(c(x, y))\}, \quad \forall x \in S,
$$

where opt denotes sup or inf, $x$ and $y$ stand for the state and decision vectors, respectively, $a, b$, and $c$ represent the transformations of the processes, and $f(x)$ represents the optimal return function with initial $x$. 
This paper is divided into four sections. In Section 2, we recall a few basic concepts and notations, establish several lemmas that will be needed further on, and suggest ten iterative algorithms for solving the functional equations (1.3)- (1.9). In Section 3, by applying the Banach fixed-point theorem, we establish the existence, uniqueness, and iterative approximations of solutions for the functional equation (1.3) in the Banach spaces $B C(S)$ and $B(S)$, respectively. By means of two iterative algorithms defined in Section 2, we obtain the existence, uniqueness, and iterative approximations of solutions for the functional equation (1.3) in the complete metric space $B B(S)$. Under certain conditions, we investigate the behaviors of solutions, nonpositive solutions, and nonnegative solutions and the convergence of iterative algorithms for the functional equations (1.3)-(1.7), respectively, in $B B(S)$. In Section 4, we construct eight nontrivial examples to explain our results, which extend and improve substantially the results due to Bellman [3], Bhakta and Choudhury [7], Liu [12], Liu and Kang [14, 15], Liu and Ume [17], Liu et al. [18], and others.

\section{Lemmas and Algorithms}

Throughout this paper, we assume that $\mathbb{R}=(-\infty,+\infty), \mathbb{R}^{+}=[0,+\infty), \mathbb{R}^{-}=(-\infty, 0], \mathbb{N}$ denotes the set of positive integers, and, for each $t \in \mathbb{R},[t]$ denotes the largest integer not exceeding $t$. Let $(X,\|\cdot\|)$ and $\left(Y,\|\cdot\|^{\prime}\right)$ be real Banach spaces, $S \subseteq X$ the state space, and $D \subseteq Y$ the decision space. Define

$$
\begin{gathered}
\Phi_{1}=\left\{\varphi: \varphi: \mathbb{R}^{+} \longrightarrow \mathbb{R}^{+} \text {is nondecreasing }\right\}, \\
\Phi_{2}=\left\{(\varphi, \psi): \varphi, \psi \in \Phi_{1}, \psi(t)>0, \lim _{n \rightarrow \infty} \psi\left(\varphi^{n}(t)\right)=0 \text { for } t>0\right\}, \\
\Phi_{3}=\left\{(\varphi, \psi): \varphi, \psi \in \Phi_{1}, \lim _{n \rightarrow \infty} \psi\left(\varphi^{n}(t)\right) \text { exists for } t>0\right\}, \\
B(S)=\{f: f: S \longrightarrow \mathbb{R} \text { is bounded }\}, \\
B C(S)=\{f: f \in B(S) \text { is continuous }\}, \\
B B(S)=\{f: f: S \longrightarrow \mathbb{R} \text { is bounded on bounded subsets of } S\} .
\end{gathered}
$$

Clearly $\left(B(S),\|\cdot\|_{1}\right)$ and $\left(B C(S),\|\cdot\|_{1}\right)$ are Banach spaces with norm $\|f\|_{1}=\sup _{x \in S}|f(x)|$.

For any $k \in \mathbb{N}$ and $f, g \in B B(S)$, let

$$
\begin{gathered}
d_{k}(f, g)=\sup \{|f(x)-g(x)|: x \in \bar{B}(0, k)\}, \\
d(f, g)=\sum_{k=1}^{\infty} \frac{1}{2^{k}} \cdot \frac{d_{k}(f, g)}{1+d_{k}(f, g)},
\end{gathered}
$$

where $\bar{B}(0, k)=\{x: x \in S$ and $\|x\| \leq k\}$. It is easy to see that $\left\{d_{k}\right\}_{k \in \mathbb{N}}$ is a countable family of pseudometrics on $B B(S)$. A sequence $\left\{x_{n}\right\}_{n \in \mathbb{N}}$ in $B B(S)$ is said to be converge to a point 
$x \in B B(S)$ if, for any $k \in \mathbb{N} d_{k}\left(x_{n}, x\right) \rightarrow 0$ as $n \rightarrow \infty$ and to be a Cauchy sequence if, for any $k \in \mathbb{N}, d_{k}\left(x_{n}, x_{m}\right) \rightarrow 0$ as $n, m \rightarrow \infty$. It is clear that $(B B(S), d)$ is a complete metric space.

Lemma 2.1. Let $\left\{a_{i}, b_{i}: 1 \leq i \leq n\right\} \subset \mathbb{R}$. Then

(a) $\operatorname{opt}\left\{a_{i}: 1 \leq i \leq n\right\}=\operatorname{opt}\left\{\operatorname{opt}\left\{a_{i}: 1 \leq i \leq n-1\right\}, a_{n}\right\}$,

(b) $\operatorname{opt}\left\{a_{i}: 1 \leq i \leq n\right\} \leq \operatorname{opt}\left\{b_{i}: 1 \leq i \leq n\right\}$ for $a_{i} \leq b_{i}, 1 \leq i \leq n$,

(c) $\max \left\{a_{i} b_{i}: 1 \leq i \leq n\right\} \leq \max \left\{a_{i}: 1 \leq i \leq n\right\} \max \left\{b_{i}: 1 \leq i \leq n\right\}$ for $\left\{a_{i}, b_{i}: 1 \leq i \leq\right.$ $n\} \subset \mathbb{R}^{+}$,

(d) $\min \left\{a_{i} b_{i}: 1 \leq i \leq n\right\} \geq \min \left\{a_{i}: 1 \leq i \leq n\right\} \min \left\{b_{i}: 1 \leq i \leq n\right\}$ for $\left\{a_{i}, b_{i}: 1 \leq i \leq n\right\} \subset$ $\mathbb{R}^{+}$,

(e) $\left|\operatorname{opt}\left\{a_{i}: 1 \leq i \leq n\right\}-\operatorname{opt}\left\{b_{i}: 1 \leq i \leq n\right\}\right| \leq \max \left\{\left|a_{i}-b_{i}\right|: 1 \leq i \leq n\right\}$.

Proof. Clearly (a)-(d) are true. Now we show (e). Note that (e) holds for $n=1$. Suppose that (e) is true for some $n \in \mathbb{N}$. It follows from (a) and Lemma 2.1 in [17] that

$$
\begin{aligned}
\mid \operatorname{opt} & \left\{a_{i}: 1 \leq i \leq n+1\right\}-\operatorname{opt}\left\{b_{i}: 1 \leq i \leq n+1\right\} \mid \\
& =\left|\operatorname{opt}\left\{\operatorname{opt}\left\{a_{i}: 1 \leq i \leq n\right\}, a_{n+1}\right\}-\operatorname{opt}\left\{\operatorname{opt}\left\{b_{i}: 1 \leq i \leq n\right\}, b_{n+1}\right\}\right| \\
& \leq \max \left\{\left|\operatorname{opt}\left\{a_{i}: 1 \leq i \leq n\right\}-\operatorname{opt}\left\{b_{i}: 1 \leq i \leq n\right\}\right|,\left|a_{n+1}-b_{n+1}\right|\right\} \\
& \leq \max \left\{\left|a_{i}-b_{i}\right|: 1 \leq i \leq n+1\right\} .
\end{aligned}
$$

Hence (e) holds for any $n \in \mathbb{N}$. This completes the proof.

Lemma 2.2. Let $\left\{a_{i}: 1 \leq i \leq n\right\} \subset \mathbb{R}$ and $\left\{b_{i}: 1 \leq i \leq n\right\} \subset \mathbb{R}^{+}$. Then

(a) $\max \left\{a_{i} b_{i}: 1 \leq i \leq n\right\} \geq \min \left\{a_{i}: 1 \leq i \leq n\right\} \max \left\{b_{i}: 1 \leq i \leq n\right\}$,

(b) $\min \left\{a_{i} b_{i}: 1 \leq i \leq n\right\} \leq \max \left\{a_{i}: 1 \leq i \leq n\right\} \min \left\{b_{i}: 1 \leq i \leq n\right\}$.

Proof. It is clear that (a) is true for $n=1$. Suppose that (a) is also true for some $n \in \mathbb{N}$. Using Lemma 2.3 in [19] and Lemma 2.1, we infer that

$$
\begin{aligned}
\max & \left\{a_{i} b_{i}: 1 \leq i \leq n+1\right\} \\
& =\max \left\{\max \left\{a_{i} b_{i}: 1 \leq i \leq n\right\}, a_{n+1} b_{n+1}\right\} \\
& \geq \max \left\{\min \left\{a_{i}: 1 \leq i \leq n\right\} \max \left\{b_{i}: 1 \leq i \leq n\right\}, a_{n+1} b_{n+1}\right\} \\
& \geq \min \left\{a_{i}: 1 \leq i \leq n+1\right\} \max \left\{b_{i}: 1 \leq i \leq n+1\right\} .
\end{aligned}
$$


That is, (a) is true for $n+1$. Therefore (a) holds for any $n \in \mathbb{N}$. Similarly we can prove (b). This completes the proof.

Lemma 2.3. Let $\left\{a_{1 n}\right\}_{n \in \mathbb{N}},\left\{a_{2 n}\right\}_{n \in \mathbb{N}}, \ldots,\left\{a_{k n}\right\}_{n \in \mathbb{N}}$ be convergent sequences in $\mathbb{R}$. Then

$$
\lim _{n \rightarrow \infty} \operatorname{opt}\left\{a_{i n}: 1 \leq i \leq k\right\}=\operatorname{opt}\left\{\lim _{n \rightarrow \infty} a_{i n}: 1 \leq i \leq k\right\}
$$

Proof. Put $\lim _{n \rightarrow \infty} a_{i n}=b_{i}$ for $1 \leq i \leq k$. In view of Lemma 2.1 we deduce that

$$
\left|\operatorname{opt}\left\{a_{\text {in }}: 1 \leq i \leq k\right\}-\operatorname{opt}\left\{b_{i}: 1 \leq i \leq k\right\}\right| \leq \max \left\{\left|a_{\text {in }}-b_{i}\right|: 1 \leq i \leq k\right\} \longrightarrow 0 \quad \text { as } n \longrightarrow \infty,
$$

which yields that

$$
\lim _{n \rightarrow \infty} \operatorname{opt}\left\{a_{i n}: 1 \leq i \leq k\right\}=\operatorname{opt}\left\{\lim _{n \rightarrow \infty} a_{i n}: 1 \leq i \leq k\right\}
$$

This completes the proof.

Lemma 2.4. (a) Assume that $A: S \times D \rightarrow \mathbb{R}$ is a mapping such that $\operatorname{opt}_{y \in D} A\left(x_{0}, y\right)$ is bounded for some $x_{0} \in S$. Then

$$
\left|\operatorname{opt}_{y \in D} A\left(x_{0}, y\right)\right| \leq \sup _{y \in D}\left|A\left(x_{0}, y\right)\right|
$$

(b) Assume that $A, B: S \times D \rightarrow \mathbb{R}$ are mappings such that $\operatorname{opt}_{y \in D} A\left(x_{1}, y\right)$ and opt $_{y \in D} B\left(x_{2}, y\right)$ are bounded for some $x_{1}, x_{2} \in S$. Then

$$
\left|\operatorname{opt}_{y \in D} A\left(x_{1}, y\right)-\underset{y \in D}{\operatorname{opt}} B\left(x_{2}, y\right)\right| \leq \sup _{y \in D}\left|A\left(x_{1}, y\right)-B\left(x_{2}, y\right)\right| .
$$

Proof. Now we show (a). If $\sup _{y \in D}\left|A\left(x_{0}, y\right)\right|=+\infty$, (a) holds clearly. Suppose that $\sup _{y \in D}\left|A\left(x_{0}, y\right)\right|<+\infty$. Note that

$$
-\left|A\left(x_{0}, y\right)\right| \leq A\left(x_{0}, y\right) \leq\left|A\left(x_{0}, y\right)\right|, \quad \forall y \in D
$$


It follows that

$$
\begin{aligned}
-\sup _{y \in D}\left|A\left(x_{0}, y\right)\right| & =\inf _{y \in D}\left\{-\left|A\left(x_{0}, y\right)\right|\right\} \leq \inf _{y \in D} A\left(x_{0}, y\right) \\
& \leq \operatorname{opt}_{y \in D} A\left(x_{0}, y\right) \leq \sup _{y \in D} A\left(x_{0}, y\right) \leq \sup _{y \in D}\left|A\left(x_{0}, y\right)\right|,
\end{aligned}
$$

which implies that

$$
\left|\operatorname{opt}_{y \in D} A\left(x_{0}, y\right)\right| \leq \sup _{y \in D}\left|A\left(x_{0}, y\right)\right|
$$

Next we show (b). If $\sup _{y \in D}\left|A\left(x_{1}, y\right)-B\left(x_{2}, y\right)\right|=+\infty$, (b) is true. Suppose that $\sup _{y \in D}\left|A\left(x_{1}, y\right)-B\left(x_{2}, y\right)\right|<+\infty$. Note that

$$
\left|A\left(x_{1}, y\right)-B\left(x_{2}, y\right)\right| \leq \sup _{y \in D}\left|A\left(x_{1}, y\right)-B\left(x_{2}, y\right)\right|<+\infty, \quad \forall y \in D
$$

which yields that

$$
\begin{aligned}
& B\left(x_{2}, y\right)-\sup _{y \in D}\left|A\left(x_{1}, y\right)-B\left(x_{2}, y\right)\right| \\
& \quad \leq A\left(x_{1}, y\right) \leq B\left(x_{2}, y\right)+\sup _{y \in D}\left|A\left(x_{1}, y\right)-B\left(x_{2}, y\right)\right|, \quad \forall y \in D .
\end{aligned}
$$

It follows that

$$
\begin{aligned}
\underset{y \in D}{\operatorname{opt}} & B\left(x_{2}, y\right)-\sup _{y \in D}\left|A\left(x_{1}, y\right)-B\left(x_{2}, y\right)\right| \\
& =\operatorname{opt}_{y \in D}\left\{B\left(x_{2}, y\right)-\sup _{y \in D}\left|A\left(x_{1}, y\right)-B\left(x_{2}, y\right)\right|\right\} \\
& \leq \operatorname{opt}_{y \in D} A\left(x_{1}, y\right) \leq \operatorname{opt}_{y \in D}\left\{B\left(x_{2}, y\right)+\sup _{y \in D}\left|A\left(x_{1}, y\right)-B\left(x_{2}, y\right)\right|\right\} \\
& =\operatorname{opt}_{y \in D} B\left(x_{2}, y\right)+\sup _{y \in D}\left|A\left(x_{1}, y\right)-B\left(x_{2}, y\right)\right|,
\end{aligned}
$$

which gives that

$$
\left|\operatorname{opt}_{y \in D} A\left(x_{1}, y\right)-\operatorname{opt}_{y \in D} B\left(x_{2}, y\right)\right| \leq \sup _{y \in D}\left|A\left(x_{1}, y\right)-B\left(x_{2}, y\right)\right|
$$

This completes the proof. 
Algorithm 1. For any $f_{0} \in B C(S)$, compute $\left\{f_{n}\right\}_{n \geq 0}$ by

$$
\begin{aligned}
& f_{n+1}(x)=\left(1-\alpha_{n}\right) f_{n}(x) \\
& \quad+\alpha_{n} \text { opt opt }\left\{p(x, y), q(x, y) f_{n}(a(x, y)),\right. \\
& \left.\quad r(x, y) f_{n}(b(x, y)), s(x, y) f_{n}(c(x, y))\right\}, \quad \forall x \in S, n \geq 0,
\end{aligned}
$$

where

$$
\left\{\alpha_{n}\right\}_{n \geq 0} \text { is any sequence in }[0,1], \quad \sum_{n=0}^{\infty} \alpha_{n}=+\infty .
$$

Algorithm 2. For any $f_{0} \in B(S)$, compute $\left\{f_{n}\right\}_{n \geq 0}$ by (2.17) and (2.18).

Algorithm 3. For any $f_{0} \in B B(S)$, compute $\left\{f_{n}\right\}_{n \geq 0}$ by (2.17) and (2.18).

Algorithm 4. For any $w_{0} \in B B(S)$, compute $\left\{w_{n}\right\}_{n \geq 0}$ by

$$
\begin{gathered}
w_{n+1}(x)=\underset{y \in D}{\operatorname{opt} o p t}\left\{p(x, y), q(x, y) w_{n}(a(x, y)), r(x, y) w_{n}(b(x, y)),\right. \\
\left.s(x, y) w_{n}(c(x, y))\right\}, \quad \forall x \in S, n \geq 0 .
\end{gathered}
$$

Algorithm 5. For any $w_{0} \in B B(S)$, compute $\left\{w_{n}\right\}_{n \geq 0}$ by

$$
\begin{gathered}
w_{n+1}(x)=\underset{y \in D}{\operatorname{opt} \max }\left\{p(x, y), q(x, y) w_{n}(a(x, y)), r(x, y) w_{n}(b(x, y)),\right. \\
\left.s(x, y) w_{n}(c(x, y))\right\}, \quad \forall x \in S, n \geq 0 .
\end{gathered}
$$

Algorithm 6. For any $w_{0} \in B B(S)$, compute $\left\{w_{n}\right\}_{n \geq 0}$ by

$$
\begin{gathered}
w_{n+1}(x)=\underset{y \in D}{\operatorname{opt} \min }\left\{p(x, y), q(x, y) w_{n}(a(x, y)), r(x, y) w_{n}(b(x, y)),\right. \\
\left.s(x, y) w_{n}(c(x, y))\right\}, \quad \forall x \in S, n \geq 0 .
\end{gathered}
$$

Algorithm 7. For any $w_{0} \in B B(S)$, compute $\left\{w_{n}\right\}_{n \geq 0}$ by

$$
\begin{gathered}
w_{n+1}(x)=\sup _{y \in D} \max \left\{p(x, y), q(x, y) w_{n}(a(x, y)), r(x, y) w_{n}(b(x, y)),\right. \\
\left.s(x, y) w_{n}(c(x, y))\right\}, \quad \forall x \in S, n \geq 0 .
\end{gathered}
$$


Algorithm 8. For any $w_{0} \in B B(S)$, compute $\left\{w_{n}\right\}_{n \geq 0}$ by

$$
\begin{gathered}
w_{n+1}(x)=\inf _{y \in D} \min \left\{p(x, y), q(x, y) w_{n}(a(x, y)), r(x, y) w_{n}(b(x, y)),\right. \\
\left.s(x, y) w_{n}(c(x, y))\right\}, \quad \forall x \in S, n \geq 0 .
\end{gathered}
$$

Algorithm 9. For any $w_{0} \in B B(S)$, compute $\left\{w_{n}\right\}_{n \geq 0}$ by

$$
\begin{gathered}
w_{n+1}(x)=\sup _{y \in D} \min \left\{p(x, y), q(x, y) w_{n}(a(x, y)), r(x, y) w_{n}(b(x, y)),\right. \\
\left.s(x, y) w_{n}(c(x, y))\right\}, \quad \forall x \in S, n \geq 0 .
\end{gathered}
$$

Algorithm 10. For any $w_{0} \in B B(S)$, compute $\left\{w_{n}\right\}_{n \geq 0}$ by

$$
\begin{gathered}
w_{n+1}(x)=\inf _{y \in D} \max \left\{p(x, y), q(x, y) w_{n}(a(x, y)), r(x, y) w_{n}(b(x, y)),\right. \\
\left.s(x, y) w_{n}(c(x, y))\right\}, \quad \forall x \in S, n \geq 0 .
\end{gathered}
$$

\section{The Properties of Solutions and Convergence of Algorithms}

Now we prove the existence, uniqueness, and iterative approximations of solutions for the functional equation (1.3) in $B C(S)$ and $B(S)$, respectively, by using the Banach fixed-point theorem.

Theorem 3.1. Let $S$ be compact. Let $p, q, r, s: S \times D \rightarrow \mathbb{R}$ and $a, b, c: S \times D \rightarrow S$ satisfy the following conditions:

(C1) $p$ is bounded in $S \times D$;

(C2) $\sup _{(x, y) \in S \times D} \max \{|q(x, y)|,|r(x, y)|,|s(x, y)|\} \leq \alpha$ for some constant $\alpha \in(0,1)$;

(C3) for each fixed $x_{0} \in S$,

$$
\begin{array}{ll}
\lim _{x \rightarrow x_{0}} p(x, y)=p\left(x_{0}, y\right), & \lim _{x \rightarrow x_{0}} q(x, y)=q\left(x_{0}, y\right), \\
\lim _{x \rightarrow x_{0}} r(x, y)=r\left(x_{0}, y\right), & \lim _{x \rightarrow x_{0}} s(x, y)=s\left(x_{0}, y\right), \\
\lim _{x \rightarrow x_{0}} a(x, y)=a\left(x_{0}, y\right), & \lim _{x \rightarrow x_{0}} b(x, y)=b\left(x_{0}, y\right), \\
\lim _{x \rightarrow x_{0}} c(x, y)=c\left(x_{0}, y\right) &
\end{array}
$$

uniformly for $y \in D$, respectively.

Then the functional equation (1.3) possesses a unique solution $f \in B C(S)$, and the sequence $\left\{f_{n}\right\}_{n \geq 0}$ generated by Algorithm 1 converges to $f$ and has the error estimate

$$
\left\|f_{n+1}-f\right\| \leq e^{-(1-\alpha) \sum_{i=0}^{n} \alpha_{i}}\left\|f_{0}-f\right\|, \quad \forall n \geq 0 .
$$


Fixed Point Theory and Applications

Proof. Define a mapping $H: B C(S) \rightarrow B C(S)$ by

$$
\begin{gathered}
H h(x)=\underset{y \in D}{\operatorname{opt} o p t}\{p(x, y), q(x, y) h(a(x, y)), r(x, y) h(b(x, y)) \\
s(x, y) h(c(x, y))\}, \quad \forall(x, h) \in S \times B C(S) .
\end{gathered}
$$

Let $h \in B C(S)$ and $x_{0} \in S$ and $\varepsilon>0$. It follows from (C1), (C3), and the compactness of $S$ that there exist constants $M>0, \delta>0$, and $\delta_{1}>0$ satisfying

$$
\begin{gathered}
\sup _{(x, y) \in S \times D}|p(x, y)| \leq M, \\
\sup _{(x, y) \in S \times D} \max \{|h(x)|,|h(a(x, y))|,|h(b(x, y))|,|h(c(x, y))|\} \leq M, \\
\left|p(x, y)-p\left(x_{0}, y\right)\right|<\frac{\varepsilon}{3}, \quad \forall(x, y) \in S \times D \text { with }\left\|x-x_{0}\right\|<\delta, \\
\max \left\{\left|q(x, y)-q\left(x_{0}, y\right)\right|,\left|r(x, y)-r\left(x_{0}, y\right)\right|,\left|s(x, y)-s\left(x_{0}, y\right)\right|\right\}<\frac{\varepsilon}{6 M}, \\
\forall(x, y) \in S \times D \quad \text { with }\left\|x-x_{0}\right\|<\delta, \\
\max \left\{\| a(x, y)-a\left(x_{1}\right)-h\left(x_{2}\right) \mid<\frac{\varepsilon}{6}, \quad \forall x_{1}, x_{2} \in S \text { with }\left\|x_{1}-x_{2}\right\|<\delta_{1},\right. \\
\left.\forall b(x, y)-b\left(x_{0}, y\right)\|,\| c(x, y)-c\left(x_{0}, y\right) \|\right\}<\delta_{1}, \\
\forall(x, y) \in S \times D \quad \text { with }\left\|x-x_{0}\right\|<\delta .
\end{gathered}
$$

Using (3.3) -(3.5), (C2), and Lemmas 2.1 and 2.4, we get that

$$
\begin{aligned}
& |H h(x)| \leq \sup _{y \in D}|\operatorname{opt}\{p(x, y), q(x, y) h(a(x, y)), r(x, y) h(b(x, y)), s(x, y) h(c(x, y))\}| \\
& \leq \sup _{y \in D}\{\max \{|p(x, y)|,|q(x, y)||h(a(x, y))| \\
& \qquad|r(x, y)||h(b(x, y))|,|s(x, y)||h(c(x, y))|\}\} \\
& \leq \sup _{y \in D}\{\max \{|p(x, y)|, \max \{|q(x, y)|,|r(x, y)|,|s(x, y)|\} \\
& \quad \times \max \{|h(a(x, y))|,|h(b(x, y))|,|h(c(x, y))|\}\} \\
& \leq \max \{M, \alpha M\} \\
& =M, \quad \forall x \in S .
\end{aligned}
$$


In light of (C2), (3.3), (3.5)-(3.9), and Lemmas 2.1 and 2.4, we deduce that for all $(x, y) \in S \times D$ with $\left\|x-x_{0}\right\|<\delta$

$$
\begin{aligned}
& \left|H h(x)-H h\left(x_{0}\right)\right| \\
& =\mid \operatorname{opt}_{y \in D}\{\operatorname{opt}\{p(x, y), q(x, y) h(a(x, y)), r(x, y) h(b(x, y)), s(x, y) h(c(x, y))\}\} \\
& \text {-opt }\left\{\operatorname{opt}\left\{p\left(x_{0}, y\right), q\left(x_{0}, y\right) h\left(a\left(x_{0}, y\right)\right), r\left(x_{0}, y\right) h\left(b\left(x_{0}, y\right)\right), s\left(x_{0}, y\right) h\left(c\left(x_{0}, y\right)\right)\right\}\right\} \mid \\
& \leq \sup _{y \in D}\left\{\operatorname { m a x } \left\{\left|p(x, y)-p\left(x_{0}, y\right)\right|,\left|q(x, y) h(a(x, y))-q\left(x_{0}, y\right) h\left(a\left(x_{0}, y\right)\right)\right|,\right.\right. \\
& \left|r(x, y) h(b(x, y))-r\left(x_{0}, y\right) h\left(b\left(x_{0}, y\right)\right)\right|, \\
& \left.\left.\left|s(x, y) h(c(x, y))-s\left(x_{0}, y\right) h\left(c\left(x_{0}, y\right)\right)\right|\right\}\right\} \\
& \leq \sup _{y \in D}\left\{\operatorname { m a x } \left\{\left|p(x, y)-p\left(x_{0}, y\right)\right|,\left|q(x, y)-q\left(x_{0}, y\right)\right||h(a(x, y))|\right.\right. \\
& +\left|q\left(x_{0}, y\right)\right|\left|h(a(x, y))-h\left(a\left(x_{0}, y\right)\right)\right|, \\
& \left|r(x, y)-r\left(x_{0}, y\right)\right||h(b(x, y))|+\left|r\left(x_{0}, y\right)\right|\left|h(b(x, y))-h\left(b\left(x_{0}, y\right)\right)\right|, \\
& \left.\left.\left|s(x, y)-s\left(x_{0}, y\right)\right||h(c(x, y))|+\left|s\left(x_{0}, y\right)\right|\left|h(c(x, y))-h\left(c\left(x_{0}, y\right)\right)\right|\right\}\right\} \\
& \leq \sup _{y \in D}\left\{\operatorname { m a x } \left\{\left|p(x, y)-p\left(x_{0}, y\right)\right|\right.\right. \\
& \max \left\{\left|q(x, y)-q\left(x_{0}, y\right)\right|,\left|r(x, y)-r\left(x_{0}, y\right)\right|,\left|s(x, y)-s\left(x_{0}, y\right)\right|\right\} \\
& \times \max \{|h(a(x, y))|,|h(b(x, y))|,|h(c(x, y))|\} \\
& +\max \left\{\left|q\left(x_{0}, y\right)\right|,\left|r\left(x_{0}, y\right)\right|,\left|s\left(x_{0}, y\right)\right|\right\} \\
& \times \max \left\{\left|h(a(x, y))-h\left(a\left(x_{0}, y\right)\right)\right|,\left|h(b(x, y))-h\left(b\left(x_{0}, y\right)\right)\right|\right. \text {, } \\
& \left.\left.\left.\left|h(c(x, y))-h\left(c\left(x_{0}, y\right)\right)\right|\right\}\right\}\right\} \\
& \leq \max \left\{\frac{\varepsilon}{3}, M \cdot \frac{\varepsilon}{6 M}+\alpha \cdot \frac{\varepsilon}{6}\right\} \\
& <\varepsilon \text {. }
\end{aligned}
$$

Thus (3.10), (3.11), and (2.17) ensure that the mapping $H: B C(S) \rightarrow B C(S)$ and Algorithm 1 are well defined.

Next we assert that the mapping $H: B C(S) \rightarrow B C(S)$ is a contraction. Let $\varepsilon>0, x \in S$, and $g, h \in B C(S)$. Suppose that opt ${ }_{y \in D}=\inf _{y \in D}$. Choose $u, v \in D$ such that

$$
\begin{gathered}
H g(x)>\operatorname{opt}\{p(x, u), q(x, u) g(a(x, u)), r(x, u) g(b(x, u)), s(x, u) g(c(x, u))\}-\varepsilon, \\
H h(x)>\operatorname{opt}\{p(x, v), q(x, v) h(a(x, v)), r(x, v) h(b(x, v)), s(x, v) h(c(x, v))\}-\varepsilon, \\
H g(x) \leq \operatorname{opt}\{p(x, v), q(x, v) g(a(x, v)), r(x, v) g(b(x, v)), s(x, v) g(c(x, v))\}, \\
H h(x) \leq \operatorname{opt}\{p(x, u), q(x, u) h(a(x, u)), r(x, u) h(b(x, u)), s(x, u) h(c(x, u))\} .
\end{gathered}
$$


Lemma 2.1 and (3.12) lead to

$$
\begin{aligned}
& |H g(x)-H h(x)| \\
& <\max \{\mid \operatorname{opt}\{p(x, u), q(x, u) g(a(x, u)), r(x, u) g(b(x, u)), s(x, u) g(c(x, u))\} \\
& -\operatorname{opt}\{p(x, u), q(x, u) h(a(x, u)), r(x, u) h(b(x, u)), s(x, u) h(c(x, u))\} \mid, \\
& \quad \operatorname{opt}\{p(x, v), q(x, v) g(a(x, v)), r(x, v) g(b(x, v)), s(x, v) g(c(x, v))\} \\
& \quad-\operatorname{opt}\{p(x, v), q(x, v) h(a(x, v)), r(x, v) h(b(x, v)), s(x, v) h(c(x, v))\} \mid\}+\varepsilon \\
& \leq \max \{\max \{|q(x, u)||g(a(x, u))-h(a(x, u))|,|r(x, u)||g(b(x, u))-h(b(x, u))|, \\
& \quad|s(x, u)||g(c(x, u))-h(c(x, u))|\}, \\
& \quad \max \{|q(x, v)||g(a(x, v))-h(a(x, v))|,|r(x, v)||g(b(x, v))-h(b(x, v))|, \\
& \quad|s(x, v)||g(c(x, v))-h(c(x, v))|\}\}+\varepsilon \\
& \leq \max \{|q(x, u)|,|r(x, u)|,|s(x, u)|,|q(x, v)|,|r(x, v)|,|s(x, v)|\}\|g-h\|_{1}+\varepsilon \\
& \leq \alpha\|g-h\|_{1}+\varepsilon,
\end{aligned}
$$

which implies that

$$
\|H g-H h\|_{1} \leq \alpha\|g-h\|_{1}+\varepsilon, \quad \forall g, h \in B C(S) .
$$

Letting $\varepsilon \rightarrow 0^{+}$in the above inequality, we know that

$$
\|H g-H h\|_{1} \leq \alpha\|g-h\|_{1}, \quad \forall g, h \in B C(S) .
$$

Similarly we conclude that (3.15) holds for opt $t_{y \in D}=\sup _{y \in D}$. The Banach fixed-point theorem yields that the contraction mapping $H$ has a unique fixed point $f \in B C(S)$. It is easy to verify that $f$ is also a unique solution of the functional equation (1.3) in $B C(S)$. By means of (2.17), (2.18), (3.15), and

$$
\begin{aligned}
& f(x)=\left(1-\alpha_{n}\right) f(x) \\
& +\quad \alpha_{n} \operatorname{opt}_{y \in D}\{\operatorname{opt}\{p(x, y), q(x, y) f(a(x, y)), \\
& \quad r(x, y) f(b(x, y)), s(x, y) f(c(x, y))\}\}, \quad \forall x \in S, n \geq 0,
\end{aligned}
$$

we infer that

$$
\begin{aligned}
\left|f_{n+1}(x)-f(x)\right| & \leq\left(1-\alpha_{n}\right)\left|f_{n}(x)-f(x)\right|+\alpha_{n}\left|H f_{n}(x)-H f(x)\right| \\
& \leq\left(1-(1-\alpha) \alpha_{n}\right)\left|f_{n}(x)-f(x)\right| \\
& \leq e^{-(1-\alpha) \sum_{i=0}^{n} \alpha_{i}}\left\|f_{0}-f\right\|_{1}, \quad \forall x \in S, n \geq 0,
\end{aligned}
$$


which yields that

$$
\left\|f_{n+1}-f\right\|_{1} \leq e^{-(1-\alpha) \sum_{i=0}^{n} \alpha_{i}}\left\|f_{0}-f\right\|_{1}, \quad \forall n \geq 0
$$

and the sequence $\left\{f_{n}\right\}_{n \geq 0}$ converges to $f$ by (2.18). This completes the proof.

Dropping the compactness of $S$ and (C3) in Theorem 3.1, we conclude immediately the following result.

Theorem 3.2. Let $p, q, r, s: S \times D \rightarrow \mathbb{R}$ and $a, b, c: S \times D \rightarrow S$ satisfy conditions (C1) and (C2). Then the functional equation (1.3) possesses a unique solution $f \in B(S)$ and the sequence $\left\{f_{n}\right\}_{n \geq 0}$ generated by Algorithm 2 converges to $f$ and satisfies (3.2).

Next we prove the existence, uniqueness, and iterative approximations of solution for the functional equation (1.3) in $B B(S)$.

Theorem 3.3. Let $p, q, r, s: S \times D \rightarrow \mathbb{R}$ and $a, b, c: S \times D \rightarrow S$ satisfy condition (C2) and the following two conditions:

(C4) $p$ is bounded on $\bar{B}(0, k) \times D$ for each $k \in \mathbb{N}$;

(C5) $\sup _{(x, y) \in \bar{B}(0, k) \times D}\{\|a(x, y)\|,\|b(x, y)\|,\|c(x, y)\|\} \leq k$ for all $k \in \mathbb{N}$.

Then the functional equation (1.3) possesses a unique solution $w \in B B(S)$, and the sequences $\left\{f_{n}\right\}_{n \geq 0}$ and $\left\{w_{n}\right\}_{n \geq 0}$ generated by Algorithms 3 and 4 , respectively, converge to $f$ and have the error estimates

$$
\begin{gathered}
d_{k}\left(f_{n+1}, w\right) \leq e^{-(1-\alpha) \sum_{i=0}^{n} \alpha_{i}} d_{k}\left(f_{0}, w\right), \quad \forall n \geq 0, k \in \mathbb{N}, \\
d_{k}\left(w_{n+1}, w\right) \leq \frac{\alpha^{n+1}}{1-\alpha} d_{k}\left(w_{1}, w_{0}\right), \quad \forall n \geq 0, \quad k \in \mathbb{N} .
\end{gathered}
$$

Proof. Define a mapping $H: B B(S) \rightarrow B B(S)$ by (3.3). Let $k \in \mathbb{N}$ and $h \in B B(S)$. Thus (C4) and (C5) guarantee that there exist $M(k)>0$ and $G(k, h)>0$ satisfying

$$
\begin{gathered}
\sup _{(x, y) \in \bar{B}(0, k) \times D}|p(x, y)| \leq M(k), \\
\sup _{(x, y) \in \bar{B}(0, k) \times D}\{|h(a(x, y))|,|h(b(x, y))|,|h(c(x, y))|\} \leq G(k, h) .
\end{gathered}
$$

Using (3.3), (3.20), (C2), (C5), and Lemmas 2.1 and 2.4, we infer that

$$
\begin{aligned}
|H h(x)| \leq \sup _{y \in D}\{\max \{|p(x, y)|,|q(x, y)||h(a(x, y))|, \\
\leq \sup _{y \in D}\{\max \{|p(x, y)|, \max \{|q(x, y)|,|r(x, y)|,|s(x, y)|\} \\
\quad \times \max \{|h(a(x, y))|,|h(b(x, y))|,|h(c(x, y))|\}\}\} \\
\leq \max \{M(k), \alpha G(k, h)\}, \quad \forall x \in \bar{B}(0, k),
\end{aligned}
$$


which means that $H$ is a self-mapping in $B B(S)$. Consequently, Algorithms 3 and 4 are well defined.

Now we claim that

$$
d_{k}(H g, H h) \leq \alpha d_{k}(g, h), \quad \forall g, h \in B B(S), k \in \mathbb{N}
$$

Let $k \in \mathbb{N}, x \in \bar{B}(0, k), g, h \in B B(S)$, and $\varepsilon>0$. Suppose that opt ${ }_{y \in D}=\inf _{y \in D}$. Select $u, v \in D$ such that (3.12) holds. Thus (3.3), (3.12), (C2), (C5), and Lemma 2.1 ensure that

$$
\begin{aligned}
& |H g(x)-H h(x)| \\
& <\max \{\mid \operatorname{opt}\{p(x, u), q(x, u) g(a(x, u)), r(x, u) g(b(x, u)), s(x, u) g(c(x, u))\} \\
& -\operatorname{opt}\{p(x, u), q(x, u) h(a(x, u)), r(x, u) h(b(x, u)), s(x, u) h(c(x, u))\} \mid \\
& \quad \operatorname{opt}\{p(x, v), q(x, v) g(a(x, v)), r(x, v) g(b(x, v)), s(x, v) g(c(x, v))\} \\
& \quad-\operatorname{opt}\{p(x, v), q(x, v) h(a(x, v)), r(x, v) h(b(x, v)), s(x, v) h(c(x, v))\} \mid\}+\varepsilon \\
& \leq \max \{\max \{|q(x, u)||g(a(x, u))-h(a(x, u))|,|r(x, u)||g(b(x, u))-h(b(x, u))| \\
& \quad|s(x, u)||g(c(x, u))-h(c(x, u))|\} \\
& \max \{|q(x, v)||g(a(x, v))-h(a(x, v))|,|r(x, v)||g(b(x, v))-h(b(x, v))| \\
& |s(x, v)||g(c(x, v))-h(c(x, v))|\}\}+\varepsilon \\
& \leq \max \{|q(x, u)|,|r(x, u)|,|s(x, u)|,|q(x, v)|,|r(x, v)|,|s(x, v)|\} d_{k}(g, h)+\varepsilon \\
& \leq \alpha d_{k}(g, h)+\varepsilon,
\end{aligned}
$$

which implies that

$$
d_{k}(H g, H h) \leq \alpha d_{k}(g, h)+\varepsilon, \quad \forall g, h \in B B(S) .
$$

Similarly we conclude that (3.24) holds for opt ${ }_{y \in D}=\sup _{y \in D}$. As $\varepsilon \rightarrow 0^{+}$in (3.24), we get that (3.22) holds.

Let $w_{0} \in B B(S)$. It follows from Algorithm 4 that

$$
w_{n+1}(x)=H w_{n}(x), \quad \forall n \geq 0, x \in S,
$$


and (3.22) leads to

$$
\begin{aligned}
d_{k}\left(w_{n+1}, w_{n+1+m}\right) & \leq \sum_{i=n+1}^{n+m} d_{k}\left(w_{i}, w_{i+1}\right)=\sum_{i=n+1}^{n+m} d_{k}\left(H w_{i-1}, H w_{i}\right) \\
& \leq \sum_{i=n+1}^{n+m} \alpha d_{k}\left(w_{i-1}, w_{i}\right) \leq \sum_{i=n+1}^{n+m} \alpha^{i} d_{k}\left(w_{0}, w_{1}\right) \\
& \leq \frac{\alpha^{n+1}}{1-\alpha} d_{k}\left(w_{0}, w_{1}\right), \quad \forall n \geq 0, k, m \in \mathbb{N},
\end{aligned}
$$

which yields that $\left\{w_{n}\right\}_{n \geq 0}$ is a Cauchy sequence in the complete metric space $(B B(S), d)$, and hence $\left\{w_{n}\right\}_{n \geq 0}$ converges to some $w \in B B(S)$. In light of (3.22) and (C2), we know that

$$
\begin{aligned}
d(H g, H h) & =\sum_{k=1}^{\infty} \frac{1}{2^{k}} \cdot \frac{d_{k}(H g, H h)}{1+d_{k}(H g, H h)} \leq \sum_{k=1}^{\infty} \frac{1}{2^{k}} \cdot \frac{\alpha d_{k}(g, h)}{1+\alpha d_{k}(g, h)} \\
& \leq \sum_{k=1}^{\infty} \frac{1}{2^{k}} \cdot \frac{\alpha d_{k}(g, h)}{\alpha+\alpha d_{k}(g, h)}=d(g, h), \quad \forall g, h \in B B(S) .
\end{aligned}
$$

That is, the mapping $H$ is nonexpansive. It follows from (3.27) and Algorithm 4 that

$$
d(H w, w)=\lim _{n \rightarrow \infty} d\left(H w_{n}, w\right)=\lim _{n \rightarrow \infty} d\left(w_{n+1}, w\right)=0,
$$

that is, $w=H w$. Suppose that there exists $u \in B B(S) \backslash\{w\}$ with $u=H u$. Consequently there exists some $k_{0} \in \mathbb{N}$ satisfying $d_{k_{0}}(w, u)>0$. It follows from (3.22) and (C2) that

$$
0<d_{k_{0}}(w, u)=d_{k_{0}}(H w, H u) \leq \alpha d_{k_{0}}(w, u)<d_{k_{0}}(w, u),
$$

which is a contradiction. Hence the mapping $H: B B(S) \rightarrow B B(S)$ has a unique fixed point $w \in B B(S)$, which is a unique solution of the functional equation (1.3) in $B B(S)$. Letting $m \rightarrow \infty$ in (3.26), we infer that

$$
d_{k}\left(w_{n+1}, w\right) \leq \frac{\alpha^{n+1}}{1-\alpha} d_{k}\left(w_{0}, w_{1}\right), \quad \forall n \geq 0, k \in \mathbb{N}
$$

It follows from Algorithm 3, (2.18), and (3.22) that

$$
\begin{aligned}
d_{k}\left(f_{n+1}, w\right) & =\sup _{x \in \bar{B}(0, k)}\left|\left(1-\alpha_{n}\right)\left(f_{n}(x)-w(x)\right)+\alpha_{n}\left(H f_{n}(x)-H w(x)\right)\right| \\
& \leq\left(1-\alpha_{n}\right) \sup _{x \in \bar{B}(0, k)}\left|f_{n}(x)-w(x)\right|+\alpha_{n} \sup _{x \in \bar{B}(0, k)}\left|H f_{n}(x)-H w(x)\right| \\
& \leq\left(1-\alpha_{n}\right) d_{k}\left(f_{n}, w\right)+\alpha_{n} d_{k}\left(H f_{n}, H w\right) \\
& \leq\left(1-(1-\alpha) \alpha_{n}\right) d_{k}\left(f_{n}, w\right) \\
& \leq e^{-(1-\alpha) \sum_{i=0}^{n} \alpha_{i}} d_{k}\left(f_{0}, w\right), \quad \forall n \geq 0, \quad k \in \mathbb{N},
\end{aligned}
$$

which gives that $f_{n} \rightarrow w$ as $n \rightarrow \infty$. This completes the proof. 
Next we investigate the behaviors of solutions for the functional equations (1.3)-(1.5) and discuss the convergence of Algorithms 4-6 in $B B(S)$, respectively.

Theorem 3.4. Let $(\varphi, \psi) \in \Phi_{2}, p, q, r, s: S \times D \rightarrow \mathbb{R}$ and $a, b, c: S \times D \rightarrow S$ satisfy the following conditions:

(C6) $\sup _{y \in D}|p(x, y)| \leq \psi(\|x\|)$ for all $x \in S$;

(C7) $\sup _{y \in D} \max \{\|a(x, y)\|,\|b(x, y)\|,\|c(x, y)\|\} \leq \varphi(\|x\|)$ for all $x \in S$;

(C8) $\sup _{(x, y) \in S \times D} \max \{|q(x, y)|,|r(x, y)|,|s(x, y)|\} \leq 1$.

Then the functional equation (1.3) possesses a solution $w \in B B(S)$ satisfying conditions (C9)-(C12) below:

(C9) the sequence $\left\{w_{n}\right\}_{n \geq 0}$ generated by Algorithm 4 converges to $w$, where $w_{0} \in B B(S)$ with $\left|w_{0}(x)\right| \leq \psi(\|x\|)$ for all $(x, k) \in \bar{B}(0, k) \times \mathbb{N} ;$

(C10) $|w(x)| \leq \psi(\|x\|)$ for all $x \in S$;

(C11) $\lim _{n \rightarrow \infty} w\left(x_{n}\right)=0$ for any $x_{0} \in S,\left\{y_{n}\right\}_{n \in \mathbb{N}} \subset D$ and $x_{n} \in\left\{a\left(x_{n-1}, y_{n}\right), b\left(x_{n-1}, y_{n}\right)\right.$, $\left.c\left(x_{n-1}, y_{n}\right)\right\}$ for all $n \in \mathbb{N}$;

(C12) $w$ is unique relative to condition (C11).

Proof. First of all we assert that

$$
\varphi(t)<t, \quad \forall t>0
$$

Suppose that there exists some $t_{0}>0$ with $\varphi\left(t_{0}\right) \geq t_{0}$. It follows from $(\varphi, \psi) \in \Phi_{2}$ that

$$
\psi\left(t_{0}\right) \leq \psi\left(\varphi\left(t_{0}\right)\right) \leq \psi\left(\varphi^{2}\left(t_{0}\right)\right) \leq \cdots \leq \psi\left(\varphi^{n}\left(t_{0}\right)\right) \longrightarrow 0 \quad \text { as } n \longrightarrow \infty
$$

That is,

$$
\psi\left(t_{0}\right) \leq 0<\psi\left(t_{0}\right)
$$

which is impossible. That is, (3.32) holds. Let the mapping $H$ be defined by (3.3) in $B B(S)$. Note that (C6) and (C7) imply (C4) and (C5) by (3.32) and $(\varphi, \psi) \in \Phi_{2}$, respectively. As in the proof of Theorem 3.3 , by (C8) we conclude that the mapping $H$ maps $B B(S)$ into $B B(S)$ and satisfies

$$
\begin{gathered}
d_{k}(H g, H h) \leq d_{k}(g, h), \quad \forall g, h \in B B(S), k \in \mathbb{N}, \\
\begin{aligned}
d(H g, H h) & =\sum_{k=1}^{\infty} \frac{1}{2^{k}} \cdot \frac{d_{k}(H g, H h)}{1+d_{k}(H g, H h)} \leq \sum_{k=1}^{\infty} \frac{1}{2^{k}} \cdot \frac{d_{k}(g, h)}{1+d_{k}(g, h)} \\
& =d(g, h), \quad \forall g, h \in B B(S) .
\end{aligned}
\end{gathered}
$$

That is, the mapping $H$ is nonexpansive. 
Let the sequence $\left\{w_{n}\right\}_{n \geq 0}$ be generated by Algorithm 4 and $w_{0} \in B B(S)$ with $\left|w_{0}(x)\right| \leq$ $\psi(\|x\|)$ for all $(x, k) \in \bar{B}(0, k) \times \mathbb{N}$. We now claim that for each $n \geq 0$

$$
\left|w_{n}(x)\right| \leq \psi(\|x\|), \quad \forall(x, k) \in \bar{B}(0, k) \times \mathbb{N} .
$$

Clearly (3.37) holds for $n=0$. Assume that (3.37) is true for some $n \geq 0$. It follows from (C6)-(C8), (3.32), Algorithm 4, and Lemmas 2.1 and 2.4 that

$$
\begin{aligned}
\left|w_{n+1}(x)\right| & =\left|\operatorname{opt}_{y \in D} \operatorname{opt}\left\{p(x, y), q(x, y) w_{n}(a(x, y)), r(x, y) w_{n}(b(x, y)), s(x, y) w_{n}(c(x, y))\right\}\right| \\
\leq & \sup _{y \in D} \max \left\{|p(x, y)|,|q(x, y)|\left|w_{n}(a(x, y))\right|\right. \\
\leq & \left.|r(x, y)|\left|w_{n}(b(x, y))\right|,|s(x, y)|\left|w_{n}(c(x, y))\right|\right\} \\
& \leq \sup _{y \in D} \max \{|p(x, y)|, \max \{|q(x, y)|,|r(x, y)|,|s(x, y)|\} \\
& \leq \sup _{y \in D} \max \left\{\psi(\|x\|), \max \left\{\psi\left(\| w_{n}(a(x, y))|,| w_{n}(b(x, y))|,| w_{n}(c(x, y)) \mid\right\}\right\}\right. \\
& \leq \max \{\psi(\|x\|), \psi(\varphi(\|x\|))\} \\
& =\psi(\|x\|) .
\end{aligned}
$$

That is, (3.37) is true for $n+1$. Hence (3.37) holds for each $n \geq 0$.

Next we claim that $\left\{w_{n}\right\}_{n \geq 0}$ is a Cauchy sequence in $(B B(S), d)$. Let $k, n, m \in \mathbb{N}, x_{0} \in$ $\bar{B}(0, k)$, and $\varepsilon>0$. Suppose that opt $\mathrm{y}_{y \in D}=\inf _{y \in D}$. Choose $y, z \in D$ with

$$
\begin{gathered}
w_{n}\left(x_{0}\right)>\operatorname{opt}\left\{p\left(x_{0}, y\right), q\left(x_{0}, y\right) w_{n-1}\left(a\left(x_{0}, y\right)\right),\right. \\
\left.r\left(x_{0}, y\right) w_{n-1}\left(b\left(x_{0}, y\right)\right), s\left(x_{0}, y\right) w_{n-1}\left(c\left(x_{0}, y\right)\right)\right\}-2^{-1} \varepsilon, \\
w_{n+m}\left(x_{0}\right)>\operatorname{opt}\left\{p\left(x_{0}, z\right), q\left(x_{0}, z\right) w_{n+m-1}\left(a\left(x_{0}, z\right)\right),\right. \\
\left.r\left(x_{0}, z\right) w_{n+m-1}\left(b\left(x_{0}, z\right)\right), s\left(x_{0}, z\right) w_{n+m-1}\left(c\left(x_{0}, z\right)\right)\right\}-2^{-1} \varepsilon, \\
w_{n}\left(x_{0}\right) \leq \operatorname{opt}\left\{p\left(x_{0}, z\right), q\left(x_{0}, z\right) w_{n-1}\left(a\left(x_{0}, z\right)\right),\right. \\
\left.r\left(x_{0}, z\right) w_{n-1}\left(b\left(x_{0}, z\right)\right), s\left(x_{0}, z\right) w_{n-1}\left(c\left(x_{0}, z\right)\right)\right\}, \\
w_{n+m}\left(x_{0}\right) \leq \operatorname{opt}\left\{p\left(x_{0}, y\right), q\left(x_{0}, y\right) w_{n+m-1}\left(a\left(x_{0}, y\right)\right),\right. \\
\left.r\left(x_{0}, y\right) w_{n+m-1}\left(b\left(x_{0}, y\right)\right), s\left(x_{0}, y\right) w_{n+m-1}\left(c\left(x_{0}, y\right)\right)\right\} .
\end{gathered}
$$


It follows from (3.39), (C8), and Lemmas 2.2 and 2.3 that

$$
\begin{aligned}
& \left|w_{n+m}\left(x_{0}\right)-w_{n}\left(x_{0}\right)\right| \\
& <\max \left\{\mid \operatorname{opt}\left\{p\left(x_{0}, y\right), q\left(x_{0}, y\right) w_{n+m-1}\left(a\left(x_{0}, y\right)\right),\right.\right. \\
& \left.r\left(x_{0}, y\right) w_{n+m-1}\left(b\left(x_{0}, y\right)\right), s\left(x_{0}, y\right) w_{n+m-1}\left(c\left(x_{0}, y\right)\right)\right\} \\
& -\operatorname{opt}\left\{p\left(x_{0}, y\right), q\left(x_{0}, y\right) w_{n-1}\left(a\left(x_{0}, y\right)\right)\right. \text {, } \\
& \left.r\left(x_{0}, y\right) w_{n-1}\left(b\left(x_{0}, y\right)\right), s\left(x_{0}, y\right) w_{n-1}\left(c\left(x_{0}, y\right)\right)\right\} \mid, \\
& \operatorname{opt}\left\{p\left(x_{0}, z\right), q\left(x_{0}, z\right) w_{n+m-1}\left(a\left(x_{0}, z\right)\right)\right. \text {, } \\
& \left.r\left(x_{0}, z\right) w_{n+m-1}\left(b\left(x_{0}, z\right)\right), s\left(x_{0}, z\right) w_{n+m-1}\left(c\left(x_{0}, z\right)\right)\right\} \\
& -\operatorname{opt}\left\{p\left(x_{0}, z\right), q\left(x_{0}, z\right) w_{n-1}\left(a\left(x_{0}, z\right)\right)\right. \text {, } \\
& \left.\left.r\left(x_{0}, z\right) w_{n-1}\left(b\left(x_{0}, z\right)\right), s\left(x_{0}, z\right) w_{n-1}\left(c\left(x_{0}, z\right)\right)\right\} \mid\right\}+2^{-1} \varepsilon \\
& \leq \max \left\{\operatorname { m a x } \left\{\left|q\left(x_{0}, y\right)\right|\left|w_{n+m-1}\left(a\left(x_{0}, y\right)\right)-w_{n-1}\left(a\left(x_{0}, y\right)\right)\right|,\right.\right. \\
& \left|r\left(x_{0}, y\right)\right|\left|w_{n+m-1}\left(b\left(x_{0}, y\right)\right)-w_{n-1}\left(b\left(x_{0}, y\right)\right)\right|, \\
& \left.\left|s\left(x_{0}, y\right)\right|\left|w_{n+m-1}\left(c\left(x_{0}, y\right)\right)-w_{n-1}\left(c\left(x_{0}, y\right)\right)\right|\right\}, \\
& \max \left\{\left|q\left(x_{0}, z\right)\right|\left|w_{n+m-1}\left(a\left(x_{0}, z\right)\right)-w_{n-1}\left(a\left(x_{0}, z\right)\right)\right|,\right. \\
& \left|r\left(x_{0}, z\right)\right|\left|w_{n+m-1}\left(b\left(x_{0}, z\right)\right)-w_{n-1}\left(b\left(x_{0}, z\right)\right)\right|, \\
& \left.\left.\left|s\left(x_{0}, z\right)\right|\left|w_{n+m-1}\left(c\left(x_{0}, z\right)\right)-w_{n-1}\left(c\left(x_{0}, z\right)\right)\right|\right\}\right\}+2^{-1} \varepsilon \\
& \leq \max \left\{\max \left\{\left|q\left(x_{0}, y\right)\right|,\left|r\left(x_{0}, y\right)\right|,\left|s\left(x_{0}, y\right)\right|\right\}\right. \\
& \times \max \left\{\left|w_{n+m-1}\left(a\left(x_{0}, y\right)\right)-w_{n-1}\left(a\left(x_{0}, y\right)\right)\right|,\left|w_{n+m-1}\left(b\left(x_{0}, y\right)\right)-w_{n-1}\left(b\left(x_{0}, y\right)\right)\right|,\right. \\
& \left.\left|w_{n+m-1}\left(c\left(x_{0}, y\right)\right)-w_{n-1}\left(c\left(x_{0}, y\right)\right)\right|\right\}, \max \left\{\left|q\left(x_{0}, z\right)\right|,\left|r\left(x_{0}, z\right)\right|,\left|s\left(x_{0}, z\right)\right|\right\} \\
& \times \max \left\{\left|w_{n+m-1}\left(a\left(x_{0}, z\right)\right)-w_{n-1}\left(a\left(x_{0}, z\right)\right)\right|,\left|w_{n+m-1}\left(b\left(x_{0}, z\right)\right)-w_{n-1}\left(b\left(x_{0}, z\right)\right)\right|,\right. \\
& \left.\left.\left|w_{n+m-1}\left(c\left(x_{0}, z\right)\right)-w_{n-1}\left(c\left(x_{0}, z\right)\right)\right|\right\}\right\}+2^{-1} \varepsilon \\
& \leq \max \left\{\left|w_{n+m-1}\left(a\left(x_{0}, y\right)\right)-w_{n-1}\left(a\left(x_{0}, y\right)\right)\right|,\right. \\
& \left|w_{n+m-1}\left(b\left(x_{0}, y\right)\right)-w_{n-1}\left(b\left(x_{0}, y\right)\right)\right|,\left|w_{n+m-1}\left(c\left(x_{0}, y\right)\right)-w_{n-1}\left(c\left(x_{0}, y\right)\right)\right|, \\
& \left|w_{n+m-1}\left(a\left(x_{0}, z\right)\right)-w_{n-1}\left(a\left(x_{0}, z\right)\right)\right|,\left|w_{n+m-1}\left(b\left(x_{0}, z\right)\right)-w_{n-1}\left(b\left(x_{0}, z\right)\right)\right|, \\
& \left.\left|w_{n+m-1}\left(c\left(x_{0}, z\right)\right)-w_{n-1}\left(c\left(x_{0}, z\right)\right)\right|\right\}+2^{-1} \varepsilon .
\end{aligned}
$$


Therefore there exist $y_{1} \in\{y, z\} \subset D$ and $x_{1} \in\left\{a\left(x_{0}, y_{1}\right), b\left(x_{0}, y_{1}\right), c\left(x_{0}, y_{1}\right)\right\}$ satisfying

$$
\left|w_{n+m}\left(x_{0}\right)-w_{n}\left(x_{0}\right)\right|<\left|w_{n+m-1}\left(x_{1}\right)-w_{n-1}\left(x_{1}\right)\right|+2^{-1} \varepsilon
$$

In a similar method, we can derive that (3.41) holds also for opt $\mathrm{y}_{y \in D}=\sup _{y \in D}$. Proceeding in this way, we choose $y_{i} \in D$ and $x_{i} \in\left\{a\left(x_{i-1}, y_{i}\right), b\left(x_{i-1}, y_{i}\right), c\left(x_{i-1}, y_{i}\right)\right\}$ for $i \in\{2,3, \ldots, n\}$ such that

$$
\begin{gathered}
\left|w_{n+m-1}\left(x_{1}\right)-w_{n-1}\left(x_{1}\right)\right|<\left|w_{n+m-2}\left(x_{2}\right)-w_{n-2}\left(x_{2}\right)\right|+2^{-2} \varepsilon \\
\left|w_{n+m-2}\left(x_{2}\right)-w_{n-2}\left(x_{2}\right)\right|<\left|w_{n+m-3}\left(x_{3}\right)-w_{n-3}\left(x_{3}\right)\right|+2^{-3} \varepsilon \\
\vdots \\
\left|w_{m+1}\left(x_{n-1}\right)-w_{1}\left(x_{n-1}\right)\right|<\left|w_{m}\left(x_{n}\right)-w_{0}\left(x_{n}\right)\right|+2^{-n} \varepsilon .
\end{gathered}
$$

On account of $(\varphi, \psi) \in \Phi_{2},(C 7),(3.37),(3.41)$, and (3.42), we gain that

$$
\begin{aligned}
\left|w_{n+m}\left(x_{0}\right)-w_{n}\left(x_{0}\right)\right| & <\left|w_{m}\left(x_{n}\right)-w_{0}\left(x_{n}\right)\right|+\sum_{i=1}^{n} 2^{-i} \varepsilon, \\
& <\left|w_{m}\left(x_{n}\right)\right|+\left|w_{0}\left(x_{n}\right)\right|+\varepsilon \\
& \leq 2 \psi\left(\left\|x_{n}\right\|\right)+\varepsilon \\
& \leq 2 \psi\left(\varphi^{n}\left(\left\|x_{0}\right\|\right)\right)+\varepsilon
\end{aligned}
$$

which yields that

$$
d_{k}\left(w_{n+m}, w_{n}\right) \leq 2 \psi\left(\varphi^{n}(k)\right)+\varepsilon
$$

Letting $\varepsilon \rightarrow 0^{+}$in the above inequality, we infer that

$$
d_{k}\left(w_{n+m}, w_{n}\right) \leq 2 \psi\left(\varphi^{n}(k)\right) .
$$

It follows from $(\varphi, \psi) \in \Phi_{2}$ and (3.45) that $\left\{w_{n}\right\}_{n \geq 0}$ is a Cauchy sequence in $(B B(S), d)$ and it converges to some $w \in B B(S)$. Algorithm 4 and (3.36) lead to

$$
\begin{aligned}
d(H w, w) & \leq d\left(H w, H w_{n}\right)+d\left(w_{n+1}, w\right) \\
& \leq d\left(w, w_{n}\right)+d\left(w_{n+1}, w\right) \longrightarrow 0 \text { as } n \longrightarrow \infty,
\end{aligned}
$$


which yields that $H w=w$. That is, the functional equation (1.3) possesses a solution $w \in$ $B B(S)$.

Now we show (C10). Let $x \in S$. Put $k=1+[\|x\|]$. It follows from (3.37), (C7), and $(\varphi, \psi) \in \Phi_{2}$ that

$$
\begin{aligned}
|w(x)| & \leq\left|w(x)-w_{n}(x)\right|+\left|w_{n}(x)\right| \\
& \leq d_{k}\left(w, w_{n}\right)+\psi(\|x\|) \longrightarrow \psi(\|x\|) \quad \text { as } n \longrightarrow \infty
\end{aligned}
$$

that is, (C10) holds.

Next we prove (C11). Given $x_{0} \in S,\left\{y_{n}\right\}_{n \in \mathbb{N}} \subset D$, and $x_{n} \in\left\{a\left(x_{n-1}, y_{n}\right), b\left(x_{n-1}, y_{n}\right)\right.$, $\left.c\left(x_{n-1}, y_{n}\right)\right\}$ for $n \in \mathbb{N}$. Put $k=\left[\left\|x_{0}\right\|\right]+1$. Note that (C7) implies that

$$
\begin{aligned}
\left\|x_{n}\right\| & \leq \max \left\{\left\|a\left(x_{n-1}, y_{n}\right)\right\|,\left\|b\left(x_{n-1}, y_{n}\right)\right\|,\left\|c\left(x_{n-1}, y_{n}\right)\right\|\right\} \\
& \leq \varphi\left(\left\|x_{n-1}\right\|\right) \leq \cdots \leq \varphi^{n}\left(\left\|x_{0}\right\|\right) \leq \varphi^{n}(k), \quad \forall n \in \mathbb{N} .
\end{aligned}
$$

In view of (3.32), (3.37), (3.48), and $(\varphi, \psi) \in \Phi_{2}$, we know that

$$
\begin{aligned}
\left|w\left(x_{n}\right)\right| & \leq\left|w\left(x_{n}\right)-w_{n}\left(x_{n}\right)\right|+\left|w_{n}\left(x_{n}\right)\right| \\
& \leq d_{k}\left(w, w_{n}\right)+\psi\left(\left\|x_{n}\right\|\right) \\
& \leq d_{k}\left(w, w_{n}\right)+\psi\left(\varphi^{n}(\|k\|)\right) \\
& \longrightarrow 0 \text { as } n \longrightarrow \infty
\end{aligned}
$$

which means that $\lim _{n \rightarrow \infty} w_{n}\left(x_{n}\right)=0$.

Finally we prove (C12). Assume that the functional equation (1.3) has another solution $h \in B B(S)$ that satisfies (C11). Let $\varepsilon>0$ and $x_{0} \in S$. Suppose that opt ${ }_{y \in D}=\inf _{y \in D}$. Select $y, z \in D$ with

$$
\begin{gathered}
w\left(x_{0}\right)>\operatorname{opt}\left\{p\left(x_{0}, y\right), q\left(x_{0}, y\right) w\left(a\left(x_{0}, y\right)\right), r\left(x_{0}, y\right) w\left(b\left(x_{0}, y\right)\right), s\left(x_{0}, y\right) w\left(c\left(x_{0}, y\right)\right)\right\}-2^{-1} \varepsilon, \\
h\left(x_{0}\right)>\operatorname{opt}\left\{p\left(x_{0}, z\right), q\left(x_{0}, z\right) h\left(a\left(x_{0}, z\right)\right), r\left(x_{0}, z\right) h\left(b\left(x_{0}, z\right)\right), s\left(x_{0}, z\right) h\left(c\left(x_{0}, z\right)\right)\right\}-2^{-1} \varepsilon, \\
w\left(x_{0}\right) \leq \operatorname{opt}\left\{p\left(x_{0}, z\right), q\left(x_{0}, z\right) w\left(a\left(x_{0}, z\right)\right), q\left(x_{0}, z\right) w\left(b\left(x_{0}, z\right)\right), r\left(x_{0}, z\right) w\left(c\left(x_{0}, z\right)\right)\right\}, \\
h\left(x_{0}\right) \leq \operatorname{opt}\left\{p\left(x_{0}, y\right), q\left(x_{0}, y\right) h\left(a\left(x_{0}, y\right)\right), r\left(x_{0}, y\right) h\left(b\left(x_{0}, y\right)\right), s\left(x_{0}, y\right) h\left(c\left(x_{0}, y\right)\right)\right\} .
\end{gathered}
$$


On account of (3.50), (C8), and Lemma 2.1, we conclude that there exist $y_{1} \in\{y, z\}$ and $x_{1} \in\left\{a\left(x_{0}, y_{1}\right), b\left(x_{0}, y_{1}\right), c\left(x_{0}, y_{1}\right)\right\}$ satisfying

$$
\begin{aligned}
& \left|w\left(x_{0}\right)-h\left(x_{0}\right)\right| \\
& <\max \left\{\mid \operatorname{opt}\left\{p\left(x_{0}, y\right), q\left(x_{0}, y\right) w\left(a\left(x_{0}, y\right)\right), r\left(x_{0}, y\right) w\left(b\left(x_{0}, y\right)\right), s\left(x_{0}, y\right) w\left(c\left(x_{0}, y\right)\right)\right\}\right. \\
& -\operatorname{opt}\left\{p\left(x_{0}, y\right), q\left(x_{0}, y\right) h\left(a\left(x_{0}, y\right)\right), r\left(x_{0}, y\right) h\left(b\left(x_{0}, y\right)\right), s\left(x_{0}, y\right) h\left(c\left(x_{0}, y\right)\right)\right\} \mid, \\
& \mid \operatorname{opt}\left\{p\left(x_{0}, z\right), q\left(x_{0}, z\right) w\left(a\left(x_{0}, z\right)\right), r\left(x_{0}, z\right) w\left(b\left(x_{0}, z\right)\right), s\left(x_{0}, z\right) w\left(c\left(x_{0}, z\right)\right)\right\} \\
& \left.\quad-\operatorname{opt}\left\{p\left(x_{0}, z\right), q\left(x_{0}, z\right) h\left(a\left(x_{0}, z\right)\right), r\left(x_{0}, z\right) h\left(b\left(x_{0}, z\right)\right), s\left(x_{0}, z\right) h\left(c\left(x_{0}, z\right)\right)\right\} \mid\right\}+2^{-1} \varepsilon \\
& \leq \max \left\{\operatorname { m a x } \left\{\left|q\left(x_{0}, y\right)\right|\left|w\left(a\left(x_{0}, y\right)\right)-h\left(a\left(x_{0}, y\right)\right)\right|,\left|r\left(x_{0}, y\right)\right|\left|w\left(b\left(x_{0}, y\right)\right)-h\left(b\left(x_{0}, y\right)\right)\right|,\right.\right. \\
& \left.\quad\left|s\left(x_{0}, y\right)\right|\left|w\left(c\left(x_{0}, y\right)\right)-h\left(c\left(x_{0}, y\right)\right)\right|\right\}, \\
& \quad \max \left\{\left|q\left(x_{0}, z\right)\right|\left|w\left(a\left(x_{0}, z\right)\right)-h\left(a\left(x_{0}, z\right)\right)\right|,\left|r\left(x_{0}, z\right)\right|\left|w\left(b\left(x_{0}, z\right)\right)-h\left(b\left(x_{0}, z\right)\right)\right|,\right. \\
& \left.\left.\quad\left|s\left(x_{0}, z\right)\right|\left|w\left(c\left(x_{0}, z\right)\right)-h\left(c\left(x_{0}, z\right)\right)\right|\right\}\right\}+2^{-1} \varepsilon \\
& \quad \max \left\{\max \left\{\left|q\left(x_{0}, y\right)\right|,\left|r\left(x_{0}, y\right)\right|,\left|s\left(x_{0}, y\right)\right|\right\}\right. \\
& \max \left\{\left|w\left(a\left(x_{0}, y\right)\right)-h\left(a\left(x_{0}, y\right)\right)\right|,\right. \\
& \left.\quad\left|w\left(b\left(x_{0}, y\right)\right)-h\left(b\left(x_{0}, y\right)\right)\right|,\left|w\left(c\left(x_{0}, y\right)\right)-w\left(c\left(x_{0}, y\right)\right)\right|\right\}, \\
& \max \left\{\left|q\left(x_{0}, z\right)\right|,\left|r\left(x_{0}, z\right)\right|,\left|s\left(x_{0}, z\right)\right|\right\} \\
& \max \left\{\left|w\left(a\left(x_{0}, z\right)\right)-h\left(a\left(x_{0}, z\right)\right)\right|,\right. \\
& \left.\left.|| w\left(b\left(x_{0}, z\right)\right)-h\left(b\left(x_{0}, z\right)\right)|,| w\left(c\left(x_{0}, z\right)\right)-h\left(c\left(x_{0}, z\right)\right) \mid\right\}\right\}+2^{-1} \varepsilon \\
& \leq \max \left\{\left|w\left(a\left(x_{0}, y\right)\right)-h\left(a\left(x_{0}, y\right)\right)\right|,\left|w\left(b\left(x_{0}, y\right)\right)-h\left(b\left(x_{0}, y\right)\right)\right|,\right. \\
& \left|w\left(c\left(x_{0}, y\right)\right)-h\left(c\left(x_{0}, y\right)\right)\right|,\left|w\left(a\left(x_{0}, z\right)\right)-h\left(a\left(x_{0}, z\right)\right)\right|, \\
& \left.\left|w\left(b\left(x_{0}, z\right)\right)-h\left(b\left(x_{0}, z\right)\right)\right|,\left|w\left(c\left(x_{0}, z\right)\right)-h\left(c\left(x_{0}, z\right)\right)\right|\right\}+2^{-1} \varepsilon
\end{aligned}
$$

that is,

$$
\left|w\left(x_{0}\right)-h\left(x_{0}\right)\right| \leq\left|w\left(x_{1}\right)-h\left(x_{1}\right)\right|+2^{-1} \varepsilon .
$$

Similarly we can prove that (3.52) holds for opt ${ }_{y \in D}=\sup _{y \in D}$. Proceeding in this way, we select $y_{i} \in D$ and $x_{i} \in\left\{a\left(x_{i-1}, y_{i}\right), b\left(x_{i-1}, y_{i}\right), c\left(x_{i-1}, y_{i}\right)\right\}$ for $i \in\{2,3, \ldots, n\}$ and $n \in \mathbb{N}$ such that

$$
\begin{aligned}
\left|w\left(x_{1}\right)-h\left(x_{1}\right)\right| & <\left|w\left(x_{2}\right)-h\left(x_{2}\right)\right|+2^{-2} \varepsilon, \\
\left|w\left(x_{2}\right)-h\left(x_{2}\right)\right| & <\left|w\left(x_{3}\right)-h\left(x_{3}\right)\right|+2^{-3} \varepsilon, \\
& \vdots \\
\left|w\left(x_{n-1}\right)-h\left(x_{n-1}\right)\right| & <\left|w\left(x_{n}\right)-h\left(x_{n}\right)\right|+2^{-n} \varepsilon .
\end{aligned}
$$


Fixed Point Theory and Applications

It follows from (3.52) and (3.53) that

$$
\left|w\left(x_{0}\right)-h\left(x_{0}\right)\right|<\left|w\left(x_{n}\right)-h\left(x_{n}\right)\right|+\varepsilon \longrightarrow \varepsilon \quad \text { as } n \longrightarrow \infty \text {. }
$$

Since $\varepsilon$ is arbitrary, we conclude immediately that $w\left(x_{0}\right)=h\left(x_{0}\right)$. This completes the proof.

Theorem 3.5. Let $(\varphi, \psi) \in \Phi_{2}, p, q, r, s: S \times D \rightarrow \mathbb{R}$ and $a, b, c: S \times D \rightarrow S$ satisfy conditions (C6)-(C8). Then the functional equation (1.4) possesses a solution $w \in B B(S)$ satisfying conditions (C10)-(C12) and the following two conditions:

(C13) the sequence $\left\{w_{n}\right\}_{n \geq 0}$ generated by Algorithm 5 converges to $w$, where $w_{0} \in B B(S)$ with $\left|w_{0}(x)\right| \leq \psi(\|x\|)$ for all $(x, k) \in \bar{B}(0, k) \times \mathbb{N} ;$

(C14) if $q, r$, and s are nonnegative and there exists a constant $\beta \in(0,1]$ such that

$$
\max \{q(x, y), r(x, y), s(x, y)\} \equiv \beta, \quad \forall(x, y) \in S \times D,
$$

then $w$ is nonnegative.

Proof. It follows from Theorem 3.4 that the functional equation (1.4) has a solution $w \in B B(S)$ that satisfies (C10)-(C13). Now we show (C14). Given $\varepsilon>0, x_{0} \in S$ and $n \in \mathbb{N}$. It follows from Lemma 2.2, (3.55), and (1.4) that there exist $y_{1} \in D$ and $x_{1} \in\left\{a\left(x_{0}, y_{1}\right), b\left(x_{0}, y_{1}\right), c\left(x_{0}, y_{1}\right)\right\}$ such that

$$
\begin{aligned}
w\left(x_{0}\right)> & \max \left\{p\left(x_{0}, y_{1}\right), q\left(x_{0}, y_{1}\right) w\left(a\left(x_{0}, y_{1}\right)\right), r\left(x_{0}, y_{1}\right) w\left(b\left(x_{0}, y_{1}\right)\right),\right. \\
& \left.s\left(x_{0}, y_{1}\right) w\left(c\left(x_{0}, y_{1}\right)\right)\right\}-2^{-1} \varepsilon \\
\geq & \max \left\{p\left(x_{0}, y_{1}\right), \max \left\{q\left(x_{0}, y_{1}\right), r\left(x_{0}, y_{1}\right), s\left(x_{0}, y_{1}\right)\right\}\right. \\
& \left.\quad \times \min \left\{w\left(a\left(x_{0}, y_{1}\right)\right), w\left(b\left(x_{0}, y_{1}\right)\right), w\left(c\left(x_{0}, y_{1}\right)\right)\right\}\right\}-2^{-1} \varepsilon \\
\geq & \max \left\{p\left(x_{0}, y_{1}\right), \beta w\left(x_{1}\right)\right\}-2^{-1} \varepsilon \\
\geq & \beta w\left(x_{1}\right)-2^{-1} \varepsilon .
\end{aligned}
$$

That is,

$$
w\left(x_{0}\right)>\beta w\left(x_{1}\right)-2^{-1} \varepsilon .
$$

Proceeding in this way, we choose $y_{i} \in D$ and $x_{i} \in\left\{a\left(x_{i-1}, y_{i}\right), b\left(x_{i-1}, y_{i}\right), c\left(x_{i-1}, y_{i}\right)\right\}$ for $i \in$ $\{2,3, \ldots, n\}$ and $n \in \mathbb{N}$ such that

$$
\begin{gathered}
w\left(x_{1}\right)>\beta w\left(x_{2}\right)-2^{-2} \beta^{-1} \varepsilon, \\
w\left(x_{2}\right)>\beta w\left(x_{3}\right)-2^{-3} \beta^{-2} \varepsilon, \\
\vdots \\
w\left(x_{n-1}\right)>\beta w\left(x_{n}\right)-2^{-n} \beta^{-n+1} \varepsilon .
\end{gathered}
$$


It follows from (3.57) and (3.58) that

$$
w\left(x_{0}\right)>\beta^{n} w\left(x_{n}\right)-\sum_{i=1}^{n} 2^{-i} \varepsilon \geq \beta^{n} w\left(x_{n}\right)-\varepsilon, \quad \forall n \in \mathbb{N} .
$$

In terms of (C8), (C11), and (3.55), we see that $\left|\beta^{n} w\left(x_{n}\right)\right| \rightarrow 0$ as $n \rightarrow \infty$. Letting $n \rightarrow \infty$ in (3.59), we get that $w\left(x_{0}\right) \geq-\varepsilon$. Since $\varepsilon>0$ is arbitrary, we infer immediately that $w\left(x_{0}\right) \geq 0$. This completes the proof.

Theorem 3.6. Let $(\varphi, \psi) \in \Phi_{3}, p, q, r, s: S \times D \rightarrow \mathbb{R}$ and $a, b, c: S \times D \rightarrow S$ satisfy conditions (C6), (C7), and the following condition:

(C15) $q, r$, and s are nonnegative and $\sup _{(x, y) \in S \times D} \max \{q(x, y), r(x, y), s(x, y)\} \leq 1$.

Then the functional equation (1.6) possesses a solution $w \in B B(S)$ satisfying $\lim _{n \rightarrow \infty} w_{n}(x)=w(x)$ for any $x \in S$, where the sequence $\left\{w_{n}\right\}_{n \geq 0}$ is generated by Algorithm 7 with $w_{0} \in B B(S), w_{0}(x) \leq \sup _{y \in D} p(x, y)$, and $\left|w_{0}(x)\right| \leq \sup _{y \in D}|p(x, y)|$ for all $x \in S$.

Proof. We are going to prove that, for any $n \in \mathbb{N}$,

$$
w_{0}(x) \leq w_{1}(x) \leq \cdots \leq w_{n}(x), \quad \forall x \in S
$$

Using $(\varphi, \psi) \in \Phi_{3}$ and Algorithm 7, we gain that

$$
\begin{aligned}
w_{0}(x) & \leq \sup _{y \in D} p(x, y) \\
& \leq \sup _{y \in D} \max \left\{p(x, y), q(x, y) w_{0}(a(x, y)), r(x, y) w_{0}(b(x, y)), s(x, y) w_{0}(c(x, y))\right\} \\
& =w_{1}(x), \quad \forall x \in S,
\end{aligned}
$$

that is, (3.60) holds for $n=1$. Assume that (3.60) holds for some $n \in \mathbb{N}$. Lemma 2.1 and (C15) lead to

$$
\begin{array}{r}
\max \left\{p(x, y), q(x, y) w_{n-1}(a(x, y)), r(x, y) w_{n-1}(b(x, y)), s(x, y) w_{n-1}(c(x, y))\right\} \\
\leq \max \left\{p(x, y), q(x, y) w_{n}(a(x, y)), r(x, y) w_{n}(b(x, y)), s(x, y) w_{n}(c(x, y))\right\} \\
\forall(x, y) \in S \times D,
\end{array}
$$


which implies that

$$
\begin{aligned}
w_{n}(x) & =\sup _{y \in D} \max \left\{p(x, y), q(x, y) w_{n-1}(a(x, y)), r(x, y) w_{n-1}(b(x, y)), s(x, y) w_{n-1}(c(x, y))\right\} \\
& \leq \sup _{y \in D} \max \left\{p(x, y), q(x, y) w_{n}(a(x, y)), r(x, y) w_{n}(b(x, y)), s(x, y) w_{n}(c(x, y))\right\} \\
& =w_{n+1}(x), \quad \forall x \in S,
\end{aligned}
$$

and hence (3.60) holds for $n+1$. That is, (3.60) holds for any $n \in \mathbb{N}$.

Now we claim that, for any $n \geq 0$,

$$
\left|w_{n}(x)\right| \leq \max \left\{\psi\left(\varphi^{i}(\|x\|)\right): 0 \leq i \leq n\right\}, \quad \forall x \in S
$$

In fact, (C6) ensures that

$$
\left|w_{0}(x)\right| \leq \sup _{y \in D}|p(x, y)| \leq \psi(\|x\|), \quad \forall x \in S
$$

that is, (3.64) is true for $n=0$. Assume that (3.64) is true for some $n \geq 0$. In view of Lemmas 2.1 and 2.4, Algorithm 7, (C6), (C7), and C(15), we gain that

$$
\begin{aligned}
&\left|w_{n+1}(x)\right| \leq \sup _{y \in D} \max \left\{|p(x, y)|, q(x, y)\left|w_{n}(a(x, y))\right|\right.\left.r(x, y)\left|w_{n}(b(x, y))\right|, s(x, y)\left|w_{n}(c(x, y))\right|\right\} \\
& \leq \sup _{y \in D} \max \{|p(x, y)|, \max \{q(x, y), r(x, y), s(x, y)\} \\
&\left.\quad \times \max \left\{\left|w_{n}(a(x, y))\right|,\left|w_{n}(b(x, y))\right|,\left|w_{n}(c(x, y))\right|\right\}\right\} \\
& \leq \sup _{y \in D} \max \left\{\psi(\|x\|), \max \left\{\psi\left(\varphi^{i}(\|a(x, y)\|)\right): 0 \leq i \leq n\right\},\right. \\
& \quad \max \left\{\psi\left(\varphi^{i}(\|b(x, y)\|)\right): 0 \leq i \leq n\right\}, \\
&\left.\quad \max \left\{\psi\left(\varphi^{i}(\|c(x, y)\|)\right): 0 \leq i \leq n\right\}\right\} \\
& \leq \max \left\{\psi(\|x\|), \max \left\{\psi\left(\varphi^{i+1}(\|x\|)\right): 0 \leq i \leq n\right\}\right\} \\
& \leq \max \left\{\psi\left(\varphi^{i}(\|x\|)\right): 0 \leq i \leq n+1\right\}, \quad \forall x \in S,
\end{aligned}
$$


which yields that (3.64) is true for $n+1$. Therefore (3.64) holds for each $n \geq 0$. Given $k \in \mathbb{N}$, note that $\lim _{n \rightarrow \infty} \psi\left(\varphi^{n}(k)\right)$ exists. It follows that there exist constants $M>0$ and $n_{0} \in \mathbb{N}$ satisfying $\psi\left(\varphi^{n}(k)\right)<M$ for any $n \geq n_{0}$. Thus (3.64) leads to

$$
\left|w_{n}(x)\right| \leq \max \left\{M, \max \left\{\psi\left(\varphi^{i}(k)\right): 0 \leq i \leq n_{0}-1\right\}\right\}, \quad \forall n \geq 0,(k, x) \in \mathbb{N} \times \bar{B}(0, k) .
$$

On account of (3.60), (3.67), and Algorithm 7, we deduce that $\left\{w_{n}(x)\right\}_{n \geq 0}$ is convergent for each $x \in S$ and $\left\{w_{n}\right\}_{n \geq 0} \in B B(S)$. Put

$$
\begin{gathered}
\lim _{n \rightarrow \infty} w_{n}(x)=w(x), \quad \forall x \in S, \\
A(x)=\sup _{y \in D} \max \{p(x, y), q(x, y) w(a(x, y)), r(x, y) w(b(x, y)), \\
s(x, y) w(c(x, y))\}, \quad \forall x \in S .
\end{gathered}
$$

Obviously (3.67) ensures that $w \in B B(S)$. Notice that

$$
\begin{aligned}
& \max \left\{p(x, y), q(x, y) w_{n-1}(a(x, y)), r(x, y) w_{n-1}(b(x, y))\right. \\
& \left.s(x, y) w_{n-1}(c(x, y))\right\} \leq w_{n}(x), \quad \forall(x, y, n) \in S \times D \times \mathbb{N} .
\end{aligned}
$$

Letting $n \rightarrow \infty$ in the above inequality, by Lemmas 2.1 and 2.3 and the convergence of $\left\{w_{n}(x)\right\}_{n \geq 0}$ we infer that

$$
\begin{array}{r}
\max \{p(x, y), q(x, y) w(a(x, y)), r(x, y) w(b(x, y)), \\
s(x, y) w(c(x, y))\} \leq w(x), \quad \forall(x, y) \in S \times D,
\end{array}
$$

which yields that

$$
\begin{aligned}
A(x) & =\sup _{y \in D} \max \{p(x, y), q(x, y) w(a(x, y)), r(x, y) w(b(x, y)), s(x, y) w(c(x, y))\} \\
& \leq w(x), \quad \forall x \in S .
\end{aligned}
$$

It follows from (3.60), (C15), and Lemma 2.1 that

$$
\begin{aligned}
& \max \left\{p(x, y), q(x, y) w_{n-1}(a(x, y)), r(x, y) w_{n-1}(b(x, y)), s(x, y) w_{n-1}(c(x, y))\right\} \\
& \leq \max \{p(x, y), q(x, y) w(a(x, y)), r(x, y) w(b(x, y)) \\
& s(x, y) w(c(x, y))\}, \quad \forall(x, y, n) \in S \times D \times \mathbb{N}
\end{aligned}
$$


which implies that

$$
\begin{aligned}
w_{n}(x)= & \sup _{y \in D} \max \left\{p(x, y), q(x, y) w_{n-1}(a(x, y)), r(x, y) w_{n-1}(b(x, y))\right. \\
& \left.s(x, y) w_{n-1}(c(x, y))\right\} \\
\leq & \sup _{y \in D} \max \{p(x, y), q(x, y) w(a(x, y)), r(x, y) w(b(x, y)), s(x, y) w(c(x, y))\} \\
= & A(x), \quad \forall(x, n) \in S \times \mathbb{N} .
\end{aligned}
$$

Letting $n \rightarrow \infty$, we gain that

$$
w(x) \leq A(x), \quad \forall x \in S
$$

It follows from (3.71) and (3.74) that $w$ is a solution of the functional equation (1.6). This completes the proof.

Following similar arguments as in the proof of Theorems 3.5 and 3.6, we have the following results.

Theorem 3.7. Let $(\varphi, \psi) \in \Phi_{2}, p, q, r, s: S \times D \rightarrow \mathbb{R}$ and $a, b, c: S \times D \rightarrow S$ satisfy conditions (C6)-(C8). Then the functional equation (1.5) possesses a solution $w \in B B(S)$ satisfying conditions (C10)-(C12) and the two following conditions:

(C16) the sequence $\left\{w_{n}\right\}_{n \geq 0}$ generated by Algorithm 6 converges to $w$, where $w_{0} \in B B(S)$ with $\left|w_{0}(x)\right| \leq \psi(\|x\|)$ for all $(x, k) \in \bar{B}(0, k) \times \mathbb{N} ;$

(C17) if $q, r$, and s are nonnegative and there exists a constant $\beta \in(0,1]$ such that

$$
\min \{q(x, y), r(x, y), s(x, y)\} \equiv \beta, \quad \forall(x, y) \in S \times D,
$$

then $w$ is nonpositive.

Theorem 3.8. Let $(\varphi, \psi) \in \Phi_{3}, p, q, r, s: S \times D \rightarrow \mathbb{R}$ and $a, b, c: S \times D \rightarrow S$ satisfy conditions (C6), (C7), and (C15). Then the functional equation (1.7) possesses a solution $w \in B B(S)$ satisfying $\lim _{n \rightarrow \infty} w_{n}(x)=w(x)$ for any $x \in S$, where the sequence $\left\{w_{n}\right\}_{n \geq 0}$ is generated by Algorithm 8 with $w_{0} \in B B(S), w_{0}(x) \geq \inf _{y \in D} p(x, y)$ and $\left|w_{0}(x)\right| \leq \sup _{y \in D}|p(x, y)|$ for all $x \in S$.

\section{Applications}

In this section we use these results in Section 3 to establish the existence of solutions, nonnegative solutions, and nonpositive solutions and iterative approximations for several functional equations, respectively. 
Example 4.1. Let $X=Y=\mathbb{R}, S=[1,2], D=\mathbb{R}^{+}$, and $\alpha=2 / 3$. It follows from Theorem 3.1 that the functional equation

$$
\begin{aligned}
f(x)=\underset{y \in D}{\operatorname{opt} \text { opt }}\{ & \left\{\frac{x^{10} y^{2}}{x+y^{2}}, \frac{\sin \left(x y^{2}\right)-\cos \left(x^{2} y\right)}{3} f\left(\frac{x+y+\sin ^{2}\left(y-y^{2}\right)}{1+y^{2}}\right),\right. \\
& \left.\frac{x+y^{2}}{(x+1)^{2}+3 y^{2}} f\left(\frac{2 x+y^{2}}{x+y^{2}}\right), \frac{x y^{2}}{2 x+3 y^{2}} f\left(\frac{2 x^{2}+y \ln (1+y)}{x^{2}+y \ln (1+y)}\right)\right\}, \quad \forall x \in S,
\end{aligned}
$$

possesses a unique solution $f \in B C(S)$ and the sequence $\left\{f_{n}\right\}_{n \geq 0}$ generated by Algorithm 1 converges to $f$ and satisfies (3.2).

Example 4.2. Let $X=Y=\mathbb{R}, S=\mathbb{R}^{+}, D=\mathbb{R}^{-}$, and $\alpha=2 / 3$. It is clear that Theorem 3.2 ensures that the functional equation

$$
\begin{aligned}
f(x)=\underset{y \in D}{\operatorname{opt} o p t}\{ & \sin ^{2}(x y) \cos \left(x^{2}-y\right), \frac{2 x}{1+3 x+\ln ^{2}(1+x-y)} f(-x y), \\
& \left.\frac{2 x^{2} y^{2} \sin \left(x^{2} y\right)}{1+3 x^{2} y^{2}} f\left(x^{2}+y^{2}\right), \frac{\cos (x+y)}{3+x-y} f\left(\left|x-y^{2}\right|\right)\right\}, \quad \forall x \in S
\end{aligned}
$$

possesses a unique solution $f \in B(S)$ and the sequence $\left\{f_{n}\right\}_{n \geq 0}$ generated by Algorithm 2 converges to $f$ and satisfies (3.2).

Remark 4.3. If $q(x, y)=r(x, y)=s(x, y), a(x, y)=b(x, y)=c(x, y)$ for all $(x, y) \in$ $S \times D$, then Theorem 3.3 reduces to a result which generalizes the result in [3, page 149] and Theorem 3.4 in [7]. The following example demonstrates that Theorem 3.3 generalizes properly the corresponding results in $[3,7]$.

Example 4.4. Let $X=Y=\mathbb{R}, S=D=\mathbb{R}^{+}$, and $\alpha=5 / 6$. It is easy to verify that Theorem 3.3 guarantees that the functional equation

$$
\begin{aligned}
& f(x)=\operatorname{opt}_{y \in D} \text { opt }\left\{\frac{x^{4}}{1+|x-y|}, \frac{2 \sin \left(x^{2}-y\right)+3 \cos \left(x-y^{2}\right)}{6+\ln (1+|x-y|)} f\left(\frac{x^{3} y^{2}}{1+x^{2} y^{2}}\right),\right. \\
& \frac{2 x^{2}+y}{1+12 x^{2}+2 y} f\left(\frac{x^{3} y \sin ^{2}\left(x^{2}+2 y-1\right)}{1+x^{2} y}\right), \\
&\left.\frac{4 \sin (x-y)-\cos \left(x^{2}-y^{2}\right)}{6+\left|\cos \left(x^{2}+y^{2}\right)\right|} f\left(\frac{x^{2} y\left|\sin \left(x^{2} y^{4}\right)\right|}{1+x y}\right)\right\}, \quad \forall x \in S,
\end{aligned}
$$

has a unique solution in $B B(S)$. However, the results in [3, page 149] and Theorem 3.4 in [7] are valid for the functional equation (4.3). 
Remark 4.5. (1) If $a(x, y)=b(x, y)=c(x, y), q(x, y)=r(x, y)=s(x, y)$ for all $(x, y) \in S \times$ $D$, then Theorems 3.4, 3.5, and 3.7 reduce to three results which generalize and unify the result in [3, page 149], Theorem 3.5 in [7], Theorem 3.5 in [12], Corollaries 2.2 and 2.3 in [14], Corollaries 3.3 and 3.4 in [17], and Theorems 2.3 and 2.4 in [18], respectively.

(2) The results in [3, page 149], Theorem 3.5 in [7], Theorem 3.5 in [12], and Theorem 3.4 in [15] are special cases of Theorem 3.5 with $q(x, y)=1, r(x, y)=s(x, y)=0$ for all $(x, y) \in S \times D$.

The examples below show that Theorems 3.4, 3.5, and 3.7 are indeed generalizations of the corresponding results in $[3,7,12,14,15,17,18]$.

Example 4.6. Let $X=Y=\mathbb{R}, S=D=\mathbb{R}^{+}$. Define two functions $\psi, \varphi: \mathbb{R}^{+} \rightarrow \mathbb{R}^{+}$by $\psi(t)=t^{2}$, $\varphi(t)=t / 2$ for all $t \in \mathbb{R}^{+}$. It is easy to see that Theorem 3.4 guarantees that the functional equation

$$
\begin{aligned}
& f(x)=\underset{y \in D}{\operatorname{opt} \text { opt }}\left\{\frac{x^{2}}{1+|x-y|}, \cos ^{3}\left(x^{2}+y^{2}\right) f\left(\frac{x^{2} y^{3}}{1+2 x y^{3}}\right)\right. \\
& \sin \left(x-y^{2}\right) f\left(\frac{x^{2} y \cos ^{2}(x-y+\ln (1+|x-y|))}{1+x^{2}+y^{2}}\right) \\
&\left.\frac{1+x^{2} y^{3}}{2+x^{2} y^{3}} f\left(\frac{x^{2} y^{2}}{1+2 x y^{2}+\cos ^{2}\left(x^{2}+y^{2}\right)}\right)\right\}, \quad \forall x \in S,
\end{aligned}
$$

possesses a solution $w \in B B(S)$ that satisfies (C9)-(C12). However, the corresponding results in $[3,7,12,14,17,18]$ are not applicable for the functional equation (4.4).

Example 4.7. Let $X=Y=\mathbb{R}=S=D$. Put $\beta=1, \psi(t)=t^{2}$, and $\varphi(t)=t / 3$ for all $t \in \mathbb{R}^{+}$. It is easy to verify that Theorem 3.5 guarantees that the functional equation

$$
\begin{aligned}
& f(x)=\underset{y \in D}{\operatorname{opt} \max }\left\{\frac{x^{3} y}{1+|x y|}, f\left(\frac{x \sin ^{2}(x-y)}{3+\ln \left(1+\sqrt{x^{2}+y^{2}}\right)}\right),\right. \\
& \\
& \qquad \frac{x^{2}+(x-y)^{2}}{1+x^{2}+(x-y)^{2}} f\left(\frac{x^{3} y^{2}}{1+3 x^{2} y^{2}+\left|x^{2}-y^{2}\right|}\right), \\
&\left.\frac{\sin ^{2}\left(x^{2}-y+1\right)}{1+x^{2}+y^{2}} f\left(\frac{x^{2} y^{4} \sin \left(x^{2}+y^{2}\right)}{1+3|x| y^{4}+\cos ^{2}(x-y)}\right)\right\}, \forall x \in S,
\end{aligned}
$$

has a solution $w \in B B(S)$ satisfying $(\mathrm{C} 10)-(\mathrm{C} 14)$. But the corresponding results in $[3,7,12$, $14,15,17,18]$ are not valid for the functional equation (4.5). 
Example 4.8. Let $X=Y=\mathbb{R}, S=D=\mathbb{R}^{+}$. Put $\psi(t)=t^{2}$ and $\varphi(t)=t$ for all $t \in \mathbb{R}^{+}$. It is easy to verify that Theorem 3.6 guarantees that the functional equation

$$
\begin{aligned}
f(x)=\sup _{y \in D} \max \{ & \frac{-x^{3} y}{1+x y}, \cos ^{2}\left(x y^{2}\right) f\left(\frac{x^{3}}{1+x^{2}+y^{2}}\right), \\
& \left(1-\sin ^{2}\left(x^{2} y \sqrt{1+\ln (1+x y)}\right)\right) f\left(x \cos ^{2}\left(x^{2}-y^{2}\right)\right), \\
& \left.\frac{\sin ^{2}(x-2 y)+\cos ^{2}(2 x-y)}{2} f\left(x \sin ^{2}\left(x^{2}+y^{2}\right)\right)\right\}, \quad \forall x \in S,
\end{aligned}
$$

has a solution $w \in B B(S)$ and the sequence $\left\{w_{n}\right\}_{n \geq 0}$ generated by Algorithm 7 satisfies that $\lim _{n \rightarrow \infty} w_{n}(x)=w(x)$ for each $x \in S$, where $w_{0} \in B B(S)$ with

$$
\begin{gathered}
w_{0}(x) \leq \sup _{y \in D} \frac{-x^{3} y}{1+x y}=0, \quad \forall x \in S, \\
\left|w_{0}(x)\right| \leq \sup _{y \in D} \frac{x^{3} y}{1+x y}=x^{2}, \quad \forall x \in S .
\end{gathered}
$$

Example 4.9. Let $X=Y=\mathbb{R}=S=D$. Put $\beta=1 / 3, \psi(t)=2 t^{4}$ and $\varphi(t)=t / 3$ for all $t \in \mathbb{R}^{+}$. It is easy to verify that Theorem 3.7 guarantees that the functional equation

$$
\begin{aligned}
& f(x)=\underset{y \in D}{\operatorname{opt} \min }\left\{x^{4}\left(\sin \left(x y^{2}\right)+\cos \left(x^{2} y\right)\right), \frac{1}{3} f\left(\frac{x^{3}}{1+3 x^{2}+|x-y|}\right),\right. \\
& \frac{1+x^{2} y^{2}}{3+x^{2} y^{2}} f\left(\frac{x^{4} \sin ^{2}\left(x^{2}-x y-y^{2}\right)}{3+|x|^{3}+\cos ^{2}\left(x^{3}-x y+y^{5}\right)}\right), \\
&\left.\frac{1+2 x^{2} y^{2}}{3+x^{2} y^{2}} f\left(\frac{x^{3} y}{1+3 x^{2}|y|+\sin ^{2}\left(x^{2}-y^{2}\right)}\right)\right\}, \quad \forall x \in S,
\end{aligned}
$$

has a solution $w \in B B(S)$ satisfying $(\mathrm{C} 10)-(\mathrm{C} 12)$, (C16), and (C17). But the corresponding results in $[3,7,12,14,17,18]$ are not valid for the functional equation (4.8). 
Example 4.10. Let $X=Y=S=\mathbb{R}, D=\mathbb{R}^{+}$. Put $\psi(t)=t /(1+t)$ and $\varphi(t)=2 t$ for all $t \in \mathbb{R}^{+}$. It is easy to verify that Theorem 3.8 guarantees that the functional equation

$$
\begin{aligned}
f(x)=\inf _{y \in D} \min \left\{\frac{x}{1+|x|(1+y)}, \frac{x^{2}+y^{2}+\sin \left(x^{2}+y^{2}\right)}{1+x^{2}+y^{2}} f\left(\frac{2 x^{2}}{1+|x|+y}\right),\right. \\
\\
\frac{|x| y+\cos ^{2}(x y)}{1+|x| y} f\left(\frac{x^{3}(1+y)\left[\sin ^{2}\left(x^{2}-y^{2}\right)+\cos ^{2}(x-y)\right]}{1+y+x^{2}(2+y)}\right), \\
\\
\frac{\left.\frac{x^{2} y^{2}}{1+x^{2} y^{2}} f\left(\frac{x^{3} y(1+1 /(1+|x| y))}{1+x^{2} y\left(1+\sin ^{2}(x y)\right)}\right)\right\}, \quad \forall x \in S,}{},
\end{aligned}
$$

possesses a solution $w \in B B(S)$ and the sequence $\left\{w_{n}\right\}_{n \geq 0}$ generated by Algorithm 8 satisfies that $\lim _{n \rightarrow \infty} w_{n}(x)=w(x)$ for each $x \in S$, where $w_{0} \in B \bar{B}(S)$ with

$$
\begin{aligned}
& w_{0}(x) \geq \inf _{y \in D} \frac{x}{1+|x|(1+y)}= \begin{cases}0, & \forall x \geq 0, \\
\frac{x}{1+|x|}, & \forall x<0,\end{cases} \\
& \left|w_{0}(x)\right| \leq \sup _{y \in D} \frac{|x|}{1+|x|(1+y)}=\frac{|x|}{1+|x|}, \quad \forall x \in S .
\end{aligned}
$$

\section{Acknowledgments}

The authors wish to thank the referees for pointing out some printing errors. This study was supported by research funds from Dong-A University.

\section{References}

[1] S. A. Belbas, "Dynamic programming and maximum principle for discrete Goursat systems," Journal of Mathematical Analysis and Applications, vol. 161, no. 1, pp. 57-77, 1991.

[2] R. Bellman, "Some functional equations in the theory of dynamic programming. I. Functions of points and point transformations," Transactions of the American Mathematical Society, vol. 80, pp. 51-71, 1955.

[3] R. Bellman, Dynamic Programming, Princeton University Press, Princeton, NJ, USA, 1957.

[4] R. Bellman, Methods of Nonlinear Analysis, Vol. II, Academic Press, New York, NY, USA, 1973.

[5] R. Bellman and E. S. Lee, "Functional equations in dynamic programming," Aequationes Mathematicae, vol. 17, no. 1, pp. 1-18, 1978.

[6] R. Bellman and M. Roosta, "A technique for the reduction of dimensionality in dynamic programming," Journal of Mathematical Analysis and Applications, vol. 88, no. 2, pp. 543-546, 1982.

[7] P. C. Bhakta and S. R. Choudhury, "Some existence theorems for functional equations arising in dynamic programming. II," Journal of Mathematical Analysis and Applications, vol. 131, no. 1, pp. 217$231,1988$.

[8] P. C. Bhakta and S. Mitra, "Some existence theorems for functional equations arising in dynamic programming," Journal of Mathematical Analysis and Applications, vol. 98, no. 2, pp. 348-346, 1984. 
[9] S. Chang and Y. H. Ma, "Coupled fixed points for mixed monotone condensing operators and an existence theorem of the solutions for a class of functional equations arising in dynamic programming," Journal of Mathematical Analysis and Applications, vol. 160, no. 2, pp. 468-479, 1991.

[10] Z. Liu, "Coincidence theorems for expansion mappings with applications to the solutions of functional equations arising in dynamic programming," Acta Scientiarum Mathematicarum, vol. 65, no. 1-2, pp. 359-369, 1999.

[11] Z. Liu, "Compatible mappings and fixed points," Acta Scientiarum Mathematicarum, vol. 65, no. 1-2, pp. 371-383, 1999.

[12] Z. Liu, "Existence theorems of solutions for certain classes of functional equations arising in dynamic programming," Journal of Mathematical Analysis and Applications, vol. 262, no. 2, pp. 529-553, 2001.

[13] Z. Liu, R. P. Agarwal, and S. M. Kang, “On solvability of functional equations and system of functional equations arising in dynamic programming," Journal of Mathematical Analysis and Applications, vol. 297, no. 1, pp. 111-130, 2004.

[14] Z. Liu and S. M. Kang, "Properties of solutions for certain functional equations arising in dynamic programming," Journal of Global Optimization, vol. 34, no. 2, pp. 273-292, 2006.

[15] Z. Liu and S. M. Kang, "Existence and uniqueness of solutions for two classes of functional equations arising in dynamic programming," Acta Mathematicae Applicatae Sinica. English Series, vol. 23, no. 2, pp. 195-208, 2007.

[16] Z. Liu and J. K. Kim, "A common fixed point theorem with applications in dynamic programming," Nonlinear Functional Analysis and Applications, vol. 11, no. 1, pp. 11-19, 2006.

[17] Z. Liu and J. S. Ume, "On properties of solutions for a class of functional equations arising in dynamic programming," Journal of Optimization Theory and Applications, vol. 117, no. 3, pp. 533-551, 2003.

[18] Z. Liu, J. S. Ume, and S. M. Kang, "Some existence theorems for functional equations arising in dynamic programming," Journal of the Korean Mathematical Society, vol. 43, no. 1, pp. 11-28, 2006.

[19] Z. Liu, Y. Xu, J. S. Ume, and S. M. Kang, "Solutions to two functional equations arising in dynamic programming," Journal of Computational and Applied Mathematics, vol. 192, no. 2, pp. 251-269, 2006.

[20] S. S. Zhang, "Some existence theorems of common and coincidence solutions for a class of systems of functional equations arising in dynamic programming," Applied Mathematics and Mechanics, vol. 12, no. 1, pp. 31-37, 1991 (Chinese).

[21] C.-L. Wang, "The principle and models of dynamic programming. II," Journal of Mathematical Analysis and Applications, vol. 135, no. 1, pp. 268-283, 1988.

[22] C.-L. Wang, "The principle and models of dynamic programming. III," Journal of Mathematical Analysis and Applications, vol. 135, no. 1, pp. 284-296, 1988.

[23] C.-L. Wang, "The principle and models of dynamic programming. V," Journal of Mathematical Analysis and Applications, vol. 137, no. 1, pp. 161-167, 1989. 